\title{
Morocco: Statistical Appendix
}

This Statistical Appendix paper for Morocco was prepared by a staff team of the International Monetary Fund as background documentation for the periodic consultation with the member country. It is based on the information available at the time it was completed on May 20, 2003. The views expressed in this document are those of the staff team and do not necessarily reflect the views of the government of Morocco or the Executive Board of the IMF.

The policy of publication of staff reports and other documents by the IMF allows for the deletion of market-sensitive information.

To assist the IMF in evaluating the publication policy, reader comments are invited and may be sent by e-mail to publicationpolicv@imf.org.

Copies of this report are available to the public from

International Monetary Fund • Publication Services

700 19th Street, N.W. $\bullet$ Washington, D.C. 20431

Telephone: (202) 6237430 • Telefax: (202) 6237201

E-mail: publications@imf.org • Internet: http://wmw.imf.org

Price: $\$ 15.00$ a copy

\section{International Monetary Fund} Washington, D.C. 


\title{
INTERNATIONAL MONETARY FUND
}

\author{
MOROCCO \\ Statistical Appendix \\ Prepared by a staff team consisting of \\ Michel Lazare (head), Domenico Fanizza, Abdourahmane Sarr, and Marwan Mikhael \\ Approved by the Middle Eastern Department
}

May 20, 2003

Contents

Page

Basic Data 3

\section{Statistical Appendix}

1. Gross Domestic Product by Sector of Origin at Current Prices, 1998-2001.............4

2. Gross Domestic Product by Sector of Origin, 1998-2002 …................................5

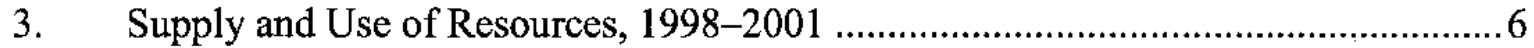

4. Savings Investment Balance, 1998-2001 ……................................................

5. Gross Fixed Capital Formation and its Components, 1998-2001 …....................... 8

6. Index of Industrial Production, 1998-2001 ……................................................

7. Foreign Direct Investment by Sector, 1998-2002 …….....................................10

8. Consumer Price, Wholesale Price, and Producer Indices, 1998-2001 .....................11

9. Evolution of Consumer Subsidies and Retail Price, 1998-2002 ……....................12

10. National Minimum Wages, July 1996-January 2003 ……................................... 13

11. Urban Population by Activity, 1998-2002 ……...............................................14

12. Financial Transactions of the Central Government, 1998-2002 .........................15

13. Central Government Operations, 1998-2002 ……........................................16

14. Central Government Revenue by Main Categories, 1998-2002 .........................18

15. Economic Classification of Central Government Expenditure, 1998-2002 ............ 19

16. Functional Classification of Central Government Current Expenditure, 1998-2002 ....................................................................2 21

17. Functional Classification of Central Government Investment Expenditure, 1998-2002 ……....................................................... 22

18. Functional Classification of Central Government

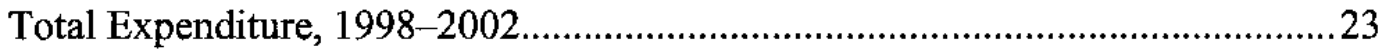

19. Administrative Classification of Central Government

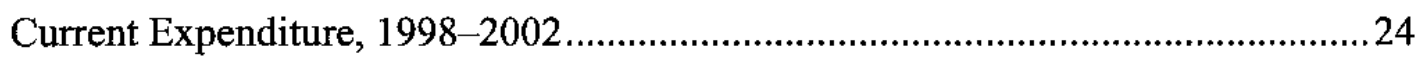

20. Administrative Classification of Central Government Investment Expenditure, 1998-2002 …………............................................25

21. Central Government Employment and Wage Indicators, 1998-2002 …...............26 
22. Central Government Debt, 1998-2002 ..........................................................27

23. External Debt Service of Central Government, 1998-2002 ..................................28

24. Current Subsidies to Public Enterprises, 1998-2002 .........................................29

25. Capital Subsidies to Public Enterprises, 1998-2002 ....................................... 30

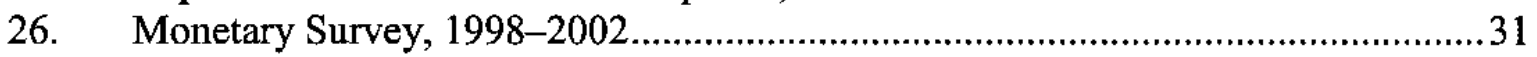

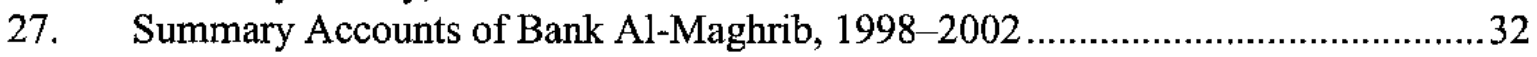

28. Summary Accounts of the Deposit Money Banks, 1998-2002 ............................33

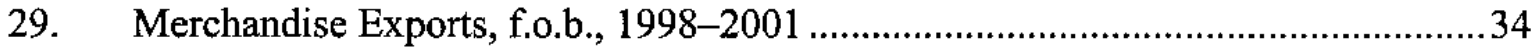

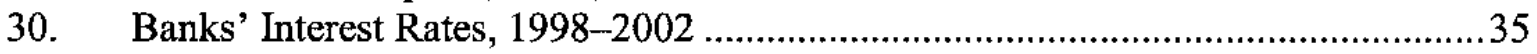

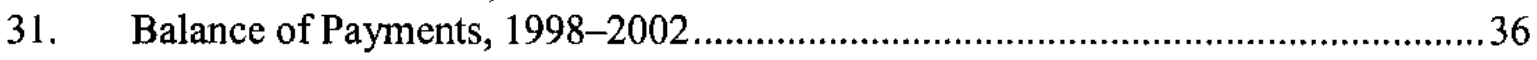

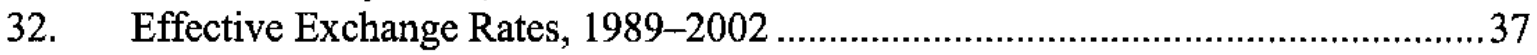

33. Stock Exchange Selected Indicators, 1998-2002 ............................................ 38

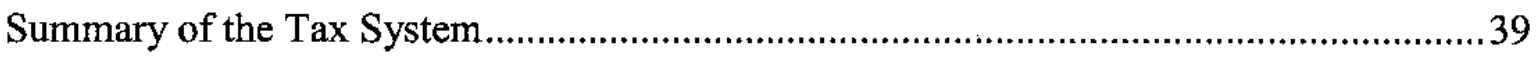




\section{Morocco: Basic Economic and Financial Indicators, 1998-2002}

Area: 459,000 square kilometers Population: Total (2002 estimate): 29.5 million

Growth rate of population (2002 estimate): 1.3 percent

GDP per capita (2002 estimate): US $\$ 1,259$

\begin{tabular}{|c|c|c|c|c|c|}
\hline & 1998 & 1999 & 2000 & 2001 & 2002 \\
\hline & \multicolumn{5}{|c|}{ (Annual percent change; unless otherwise indicated) } \\
\hline \multicolumn{6}{|l|}{ Production and income } \\
\hline Nominal GDP & 8.1 & 0.5 & 2.5 & 8.1 & 6.9 \\
\hline Real GDP & 7.7 & -0.1 & 1.0 & 6.5 & 4.5 \\
\hline Real non-agricultural GDP & 4.4 & 3.2 & 3.5 & 3.7 & 3.9 \\
\hline GDP deflator & 0.4 & 0.5 & 1.5 & 1.6 & 2.4 \\
\hline \multirow[t]{2}{*}{ Consumer price index (CPI), average } & 2.7 & 0.7 & 1.9 & 0.6 & 2.8 \\
\hline & \multicolumn{5}{|c|}{ (In billions of U.S. dollars; unless otherwise indicated) } \\
\hline \multicolumn{6}{|l|}{ External sector } \\
\hline Exports of goods, f.o.b. & 7.1 & 7.5 & 7.4 & 7.1 & 7.7 \\
\hline Imports of goods, f.o.b. & 9.5 & 10.0 & 10.7 & 10.2 & 10.7 \\
\hline Net services & -0.2 & 0.1 & 0.3 & 1.1 & 0.4 \\
\hline Net transfers & 2.3 & 2.1 & 2.4 & 3.5 & 3.6 \\
\hline Current account (in percent of GDP) & -0.4 & -0.5 & -1.4 & 4.8 & 2.9 \\
\hline \multirow[t]{2}{*}{ Overall balance (deficit -) } & 0.2 & 1.6 & -0.4 & 3.8 & 0.6 \\
\hline & \multicolumn{5}{|c|}{ (In percent of GDP) } \\
\hline \multicolumn{6}{|l|}{ Central government } \\
\hline Revenue, excluding grants and privatization & 27.2 & 26.9 & 26.2 & 24.9 & 24.0 \\
\hline Total expenditure (including Fonds Hassan II) & 29.8 & 31.4 & 32.4 & 31.1 & 29.1 \\
\hline Overall balance & -2.6 & -4.5 & -6.4 & -5.7 & -4.5 \\
\hline Privatization and GSM receipts & 0.1 & 3.2 & 0.0 & 6.1 & 0.2 \\
\hline Overall balance, incl. privatization & -2.5 & -1.3 & -6.4 & 0.4 & -4.3 \\
\hline \multicolumn{6}{|l|}{ Official reserves } \\
\hline Gross official reserves (in billions of dollars, end-period) & 4.6 & 5.7 & 4.8 & 8.4 & 10.1 \\
\hline In months of imports of goods, c.i.f. & 4.8 & 5.7 & 4.6 & 8.2 & 9.4 \\
\hline \multicolumn{6}{|l|}{ Debt (short-, medium-, and long-term) } \\
\hline Total external debt (in billions of dollars) & 20.6 & 19.8 & 18.0 & 15.9 & 16.2 \\
\hline Total extemal debt (in percent of GDP) & 57.5 & 56.1 & 53.9 & 47.0 & 43.7 \\
\hline Domestic government debt (in percent of GDP) $1 /$ & 38.1 & 41.3 & 42.2 & 45.9 & 47.9 \\
\hline Total government debt (in percent of GDP) $1 /$ & 74.3 & 76.9 & 76.4 & 74.8 & 70.7 \\
\hline \multicolumn{6}{|l|}{ Memorandum items: } \\
\hline GDP at current prices (in billions of Dh) & 344.0 & 345.6 & 354.1 & 382.9 & 409.4 \\
\hline
\end{tabular}

Sources: Data provided by the Moroccan authorities; includes Fund staff projections.

1/ Remunerated debt only. 
Table 1. Morocco: Gross Domestic Product by Sector of Origin

at Current Prices, 1998-2001

\begin{tabular}{|c|c|c|c|c|}
\hline & 1998 & 1999 & 2000 & 2001 \\
\hline & \multicolumn{4}{|c|}{ (In millions of Moroccan dirhams) } \\
\hline $\begin{array}{l}\text { Primary sector } \\
\text { Agriculture, livestock, and fishing }\end{array}$ & $\begin{array}{l}59,211 \\
59,211\end{array}$ & $\begin{array}{l}52,905 \\
52,905\end{array}$ & $\begin{array}{l}49,570 \\
49,570\end{array}$ & $\begin{array}{l}60,546 \\
60,546\end{array}$ \\
\hline $\begin{array}{l}\text { Secondary sector } \\
\text { Mining } \\
\text { Energy and water } \\
\text { Manufacturing } \\
\text { Construction and public works }\end{array}$ & $\begin{array}{r}108,669 \\
7,335 \\
27,462 \\
58,400 \\
15,472\end{array}$ & $\begin{array}{r}110,552 \\
7,464 \\
27,159 \\
59,582 \\
16,348\end{array}$ & $\begin{array}{r}112,867 \\
7,036 \\
25,264 \\
62,267 \\
18,300\end{array}$ & $\begin{array}{r}118,238 \\
7,446 \\
26,589 \\
64,851 \\
19,352\end{array}$ \\
\hline $\begin{array}{l}\text { Tertiary sector } \\
\text { Commerce } \\
\text { Transport and communications } \\
\text { Other services } \\
\text { Indirect taxes less subsidies }\end{array}$ & $\begin{array}{r}128,891 \\
39,312 \\
19,796 \\
42,992 \\
26,792\end{array}$ & $\begin{array}{r}132,713 \\
39,843 \\
21,678 \\
44,695 \\
26,496\end{array}$ & $\begin{array}{r}141,142 \\
42,549 \\
24,700 \\
45,759 \\
28,134\end{array}$ & $\begin{array}{r}145,974 \\
44,533 \\
26,367 \\
46,713 \\
28,362\end{array}$ \\
\hline Government & 47,234 & 49,424 & 50,489 & 58,138 \\
\hline \multirow[t]{2}{*}{ Gross domestic product (GDP) } & 344,005 & 345,594 & 354,068 & 382,897 \\
\hline & \multicolumn{4}{|c|}{ (Percentage change) } \\
\hline $\begin{array}{l}\text { Primary sector } \\
\text { Agriculture, livestock, and fishing }\end{array}$ & $\begin{array}{l}20.5 \\
20.5\end{array}$ & $\begin{array}{l}-10.6 \\
-10.6\end{array}$ & $\begin{array}{l}-6.3 \\
-6.3\end{array}$ & $\begin{array}{l}22.1 \\
22.1\end{array}$ \\
\hline $\begin{array}{l}\text { Secondary sector } \\
\text { Mining } \\
\text { Energy and water } \\
\text { Manufacturing } \\
\text { Construction and public works }\end{array}$ & $\begin{array}{l}3.5 \\
2.8 \\
2.2 \\
3.9 \\
4.8\end{array}$ & $\begin{array}{r}1.7 \\
1.8 \\
-1.1 \\
2.0 \\
5.7\end{array}$ & $\begin{array}{r}2.1 \\
-5.7 \\
-7.0 \\
4.5 \\
11.9\end{array}$ & $\begin{array}{l}4.8 \\
5.8 \\
5.2 \\
4.1 \\
5.7\end{array}$ \\
\hline $\begin{array}{l}\text { Tertiary sector } \\
\text { Commerce } \\
\text { Transport and communications } \\
\text { Other services } \\
\text { Indirect taxes less subsidies }\end{array}$ & $\begin{array}{l}6.7 \\
7.2 \\
6.7 \\
6.9 \\
5.5\end{array}$ & $\begin{array}{r}3.0 \\
1.4 \\
9.5 \\
4.0 \\
-1.1\end{array}$ & $\begin{array}{r}6.4 \\
6.8 \\
13.9 \\
2.4 \\
6.2\end{array}$ & $\begin{array}{l}3.4 \\
4.7 \\
6.7 \\
2.1 \\
0.8\end{array}$ \\
\hline Government & 8.9 & 4.6 & 2.2 & 15.1 \\
\hline \multirow[t]{2}{*}{ Gross domestic product (GDP) } & 8.1 & 0.5 & 2.5 & 8.1 \\
\hline & \multicolumn{4}{|c|}{ (Percentage of GDP) } \\
\hline $\begin{array}{l}\text { Primary sector } \\
\text { Agriculture, livestock, and fishing }\end{array}$ & $\begin{array}{l}17.2 \\
17.2\end{array}$ & $\begin{array}{l}15.3 \\
15.3\end{array}$ & $\begin{array}{l}14.0 \\
14.0\end{array}$ & $\begin{array}{l}15.8 \\
15.8\end{array}$ \\
\hline $\begin{array}{l}\text { Secondary sector } \\
\text { Mining } \\
\text { Energy and water } \\
\text { Manufacturing } \\
\text { Construction and public works }\end{array}$ & $\begin{array}{r}31.6 \\
2.1 \\
8.0 \\
17.0 \\
4.5\end{array}$ & $\begin{array}{r}32.0 \\
2.2 \\
7.9 \\
17.2 \\
4.7\end{array}$ & $\begin{array}{r}31.9 \\
2.0 \\
7.1 \\
17.6 \\
5.2\end{array}$ & $\begin{array}{r}30.9 \\
1.9 \\
6.9 \\
16.9 \\
5.1\end{array}$ \\
\hline $\begin{array}{l}\text { Tertiary sector } \\
\text { Commerce } \\
\text { Transport and communications } \\
\text { Other services } \\
\text { Indirect taxes less subsidies }\end{array}$ & $\begin{array}{r}37.5 \\
11.4 \\
5.8 \\
12.5 \\
7.8\end{array}$ & $\begin{array}{r}38.4 \\
11.5 \\
6.3 \\
12.9 \\
7.7\end{array}$ & $\begin{array}{r}39.9 \\
12.0 \\
7.0 \\
12.9 \\
7.9\end{array}$ & $\begin{array}{r}38.1 \\
11.6 \\
6.9 \\
12.2 \\
7.4\end{array}$ \\
\hline Government & 13.7 & 14.3 & 14.3 & 15.2 \\
\hline Gross domestic product (GDP) & 100.0 & 100.0 & 100.0 & 100.0 \\
\hline
\end{tabular}

Source: Ministry of Economic Forecast and Planning. 
Table 2. Morocco: Gross Domestic Product by Sector of Origin, 1998-2002

\begin{tabular}{|c|c|c|c|c|c|}
\hline & 1998 & 1999 & 2000 & 2001 & 2002 \\
\hline & \multicolumn{5}{|c|}{ (In millions of Moroccan dirhams at 1980 prices) } \\
\hline Primary sector & 21,901 & 18,251 & 15,577 & 19,823 & 21,413 \\
\hline Agriculture, livestock, and fishing & 21,901 & 18,251 & 15,577 & 19,823 & 21,413 \\
\hline Secondary sector & 39,358 & 40,422 & 41,448 & 43,668 & 45,414 \\
\hline Mining & 3,951 & 3,905 & 3,701 & 3,977 & 4,148 \\
\hline Energy and water & 6,544 & 6,754 & 6,722 & 7,265 & 7,547 \\
\hline Manufacturing & 23,277 & 23,906 & 24,749 & 25,785 & 26,714 \\
\hline Construction and public works & 5,586 & 5,857 & 6,276 & 6,641 & 7,007 \\
\hline Tertiary sector & 50,005 & 51,924 & 54,553 & 55,729 & 57,743 \\
\hline Commerce & 16,467 & 16,779 & 17,459 & 18,365 & 19,154 \\
\hline Transport and communications & 8,655 & 9,358 & 10,234 & 10,333 & 10,746 \\
\hline Other services & 14,795 & 14,948 & 15,157 & 15,260 & 15,658 \\
\hline Indirect taxes less subsidies & 10,088 & 10,839 & 11,703 & 11,773 & 12,185 \\
\hline Government & 22,465 & 23,027 & 23,351 & 24,428 & 25,503 \\
\hline \multirow[t]{2}{*}{ Gross domestic product (GDP) } & 133,729 & 133,623 & 134,929 & 143,648 & 150,073 \\
\hline & \multicolumn{5}{|c|}{ (Annual percentage change) } \\
\hline Primary sector & 27.9 & -16.7 & -14.6 & 27.3 & 8.0 \\
\hline Agriculture, livestock, and fishing & 27.9 & -16.7 & -14.6 & 27.3 & 8.0 \\
\hline Secondary sector & 2.3 & 2.7 & 2.5 & 5.4 & 4.0 \\
\hline Mining & -3.3 & -1.2 & -5.2 & 7.5 & 4.3 \\
\hline Energy and water & 5.1 & 3.2 & -0.5 & 8.1 & 3.9 \\
\hline Manufacturing & 2.1 & 2.7 & 3.5 & 4.2 & 3.6 \\
\hline Construction and public works & 4.1 & 4.9 & 7.2 & 5.8 & 5.5 \\
\hline Tertiary sector & 6.8 & 3.8 & 5.1 & 2.2 & 3.6 \\
\hline Commerce & 7.5 & 1.9 & 4.1 & 5.2 & 4.3 \\
\hline Transport and communications & 8.7 & 8.1 & 9.4 & 1.0 & 4.0 \\
\hline Other services & 4.2 & 1.0 & 1.4 & 0.7 & 2.6 \\
\hline Import duties less subsidies & 7.9 & 7.4 & 8.0 & 0.6 & 3.5 \\
\hline Government & 3.2 & 2.5 & 1.4 & 4.6 & 4.4 \\
\hline Gross domestic product (GDP) & 7.7 & -0.1 & 1.0 & 6.5 & 4.5 \\
\hline
\end{tabular}

Source: Ministry of Economic Forecast and Planning. 
Table 3. Morocco: Supply and Use of Resources, 1998-2001

\begin{tabular}{|c|c|c|c|c|}
\hline & 1998 & 1999 & 2000 & 2001 \\
\hline & \multicolumn{4}{|c|}{ (In millions of Moroccan dirhams) } \\
\hline Gross domestic product & 344,005 & 345,594 & 354,068 & 382,897 \\
\hline Imports of goods and nonfactor services $1 / 2 /$ & 89,433 & 95,110 & 111,129 & 115,554 \\
\hline Total resources & 433,438 & 440,704 & 465,197 & 498,451 \\
\hline Gross domestic expenditure & 372,474 & 375,153 & 393,337 & 421,849 \\
\hline Domestic consumption 3/ & 296,257 & 295,241 & 309,984 & 334,454 \\
\hline Nongovernment & 234,256 & 229,095 & 242,295 & 259,835 \\
\hline Government & 62,001 & 66,146 & 67,689 & 74,619 \\
\hline Gross fixed investment & 75,739 & 81,896 & 84,982 & 85,264 \\
\hline Nongovernment & 66,307 & 71,872 & 74,186 & 74,103 \\
\hline Government & 9,432 & 10,024 & 10,796 & 11,161 \\
\hline Change in stocks & 478 & $-1,984$ & $-1,629$ & 2,131 \\
\hline Exports of goods and nonfactor services $2 / 4 /$ & 60,964 & 65,551 & 71,860 & 76,602 \\
\hline \multicolumn{5}{|l|}{ Memorandum items: } \\
\hline Net income from abroad (including transfers) & 14,402 & 13,243 & 16,981 & 32,767 \\
\hline \multirow[t]{2}{*}{ National disposable income } & 358,407 & 358,837 & 371,049 & 415,664 \\
\hline & \multicolumn{4}{|c|}{ (In percent of GDP) } \\
\hline Gross domestic product & 100.0 & 100.0 & 100.0 & 100.0 \\
\hline Imports of goods and nonfactor services $1 / 2 /$ & 26.1 & 27.7 & 31.2 & 30.2 \\
\hline Total resources & 126.1 & 127.7 & 131.2 & 130.2 \\
\hline Gross domestic expenditure & 108.3 & 108.6 & 110.8 & 110.2 \\
\hline Domestic consumption 3/ & 85.8 & 84.4 & 85.6 & 87.3 \\
\hline Nongovernment & 67.7 & 65.1 & 66.2 & 67.9 \\
\hline Government & 18.1 & 19.3 & 19.4 & 19.5 \\
\hline Gross fixed investment & 22.4 & 24.3 & 25.0 & 22.3 \\
\hline Nongovernment & 19.6 & 21.4 & 21.9 & 19.4 \\
\hline Government & 2.8 & 2.9 & 3.1 & 2.9 \\
\hline Change in stocks & 0.1 & -0.2 & 0.2 & 0.6 \\
\hline Exports of goods and nonfactor services $2 / 4$ / & 17.8 & 19.1 & 20.4 & 20.0 \\
\hline \multicolumn{5}{|l|}{ Memorandum items: } \\
\hline Net income from abroad (including transfers) & 4.2 & 3.9 & 4.8 & 8.6 \\
\hline National disposable income & 104.2 & 103.9 & 104.8 & 108.6 \\
\hline
\end{tabular}

Source: Ministry of Economic Forecast and Planning.

1/ Excludes consumption of residents abroad.

2/ Exports and imports include goods traded under the régime d'admission temporaire sans paiement.

3 / Local consumption of residents and nonresidents (excludes consumption of residents abroad).

4/ Excludes consumption of nonresidents in Morocco. 
Table 4. Morocco: Savings-Investment Balance, 1998-2001

\begin{tabular}{|c|c|c|c|c|}
\hline & 1998 & 1999 & 2000 & 2001 \\
\hline & \multicolumn{4}{|c|}{ (In millions of Moroccan dirhams) } \\
\hline Gross national product & 358,407 & 358,837 & 371,049 & 415,664 \\
\hline National consumption $1 /$ & 283,570 & 280,562 & 292,573 & 309,627 \\
\hline Nongovernment & 221,569 & 214,416 & 224,884 & 235,008 \\
\hline Government & 62,001 & 66,146 & 67,689 & 74,619 \\
\hline Gross national savings & 74,837 & 78,275 & 78,476 & 106,037 \\
\hline Nongovernment & 68,939 & 72,314 & 76,057 & 102,722 \\
\hline Government & 5,898 & 5,961 & 2,419 & 3,315 \\
\hline Gross investment & 76,217 & 79,912 & 83,353 & 87,395 \\
\hline Gross fixed investment & 75,739 & 81,896 & 84,982 & 85,264 \\
\hline Nongovernment & 66,307 & 71,872 & 74,186 & 74,103 \\
\hline Government & 9,432 & 10,024 & 10,796 & 11,161 \\
\hline Changes in stocks & 478 & $-1,984$ & $-1,629$ & 2,131 \\
\hline National saving-investment gap & $-1,380$ & $-1,637$ & $-4,877$ & 18,642 \\
\hline Nongovernment & 2,154 & 2,426 & 3,499 & 26,488 \\
\hline Government & $-3,534$ & $-4,063$ & $-8,377$ & $-7,846$ \\
\hline Capital transfers & -96 & -84 & -63 & -101 \\
\hline Saving-investment gap, including capital transfers & $-1,476$ & $-1,721$ & $-4,940$ & 18,541 \\
\hline External current account (national accounts data) & $-1,476$ & $-1,721$ & $-5,271$ & 18,541 \\
\hline Exports of goods and nonfactor services $2 / 3 /$ & 77,719 & 84,663 & 93,504 & 105,435 \\
\hline Imports of goods and nonfactor services $3 / 4 /$ & $-93,500$ & $-99,543$ & $-115,693$ & $-119,560$ \\
\hline Net income from abroad (including transfers) & 14,402 & 13,243 & 16,981 & 32,767 \\
\hline \multirow[t]{2}{*}{ Capital transfers } & -96 & -84 & -63 & -101 \\
\hline & \multicolumn{4}{|c|}{ (In percent of GDP) } \\
\hline Gross national product & 104.2 & 103.8 & 104.8 & 108.6 \\
\hline National consumption $1 /$ & 82.4 & 81.2 & 82.6 & 80.9 \\
\hline Nongovernment & 64.4 & 62.0 & 63.5 & 61.4 \\
\hline Government & 18.0 & 19.1 & 19.1 & 19.5 \\
\hline Gross national savings & 21.8 & 22.6 & 22.2 & 27.7 \\
\hline Nongovernment & 20.0 & 20.9 & 21.5 & 26.8 \\
\hline Government & 1.7 & 1.7 & 0.7 & 0.9 \\
\hline Gross investment & 22.2 & 23.1 & 23.5 & 22.8 \\
\hline Nongovernment & 19.4 & 20.2 & 20.5 & 19.9 \\
\hline Government & 2.7 & 2.9 & 3.0 & 2.9 \\
\hline National savings-investment gap & -0.4 & -0.5 & -1.4 & 4.9 \\
\hline Nongovernment & 0.6 & 0.7 & 1.0 & 6.9 \\
\hline Government & -1.0 & -1.2 & -2.4 & -2.0 \\
\hline Capital transfers & 0.0 & 0.0 & 0.0 & 0.0 \\
\hline External current account (national accounts data) & -0.4 & -0.5 & -1.3 & 4.5 \\
\hline \multicolumn{5}{|l|}{ Memorandum items: } \\
\hline Exports of goods and nonfactor services $2 / 3 /$ & 21.7 & 23.6 & 25.2 & 25.4 \\
\hline Imports of goods and nonfactor services $3 / 4$ / & -26.1 & -27.7 & -31.2 & -28.8 \\
\hline
\end{tabular}

Source: Ministry of Economic Forecast and Planning.

1/ Consumption of residents (including consumption of residents abroad but excluding local consumption of nonresidents).

$2 /$ Including local consumption of nonresidents.

3/ From 1994 exports and imports including goods traded under the régime d'admission temporaire sans paiement.

4/ Including consumption of residents abroad. 
Table 5. Morocco: Gross Fixed Capital Formation and its Components, 1998-2001

\begin{tabular}{|c|c|c|c|c|}
\hline & 1998 & 1999 & 2000 & 2001 \\
\hline & \multicolumn{4}{|c|}{ (In millions of Moroccan dirhams at current prices) } \\
\hline Machinery and equipment & 40,359 & 45,266 & 45,266 & 40010 \\
\hline Construction & 20,828 & 22,075 & 22,892 & 24428 \\
\hline Public works & 11,915 & 12,583 & 15,666 & 16861 \\
\hline Land management, forestation, and livestock & 3,551 & 3,565 & 3,611 & 3965 \\
\hline \multicolumn{5}{|l|}{ Of which: } \\
\hline Government agencies & 478 & 10,024 & 10,796 & 11,161 \\
\hline \multirow[t]{2}{*}{ In percent of total } & 1 & 14 & 15 & 13.1 \\
\hline & \multicolumn{4}{|c|}{ (In percent of GDP) } \\
\hline Machinery and equipment & 11.5 & 12.6 & 12.0 & 10.4 \\
\hline Construction & 6.1 & 6.4 & 6.5 & 6.4 \\
\hline Public works & 3.5 & 3.6 & 4.4 & 4.4 \\
\hline Land management, forestation, and livestock & 1.0 & 1.0 & 1.1 & 1.0 \\
\hline Gross fixed capital formation & 22.0 & 23.7 & 24.0 & 22.3 \\
\hline
\end{tabular}

Source: Ministry of Economic Forecast and Planning. 
Table 6. Morocco: Index of Industrial Production, 1998-2001

$$
(1992=100 ; \text { period average })
$$

\begin{tabular}{lrrrrr}
\hline & Weights & 1998 & 1999 & 2000 & 2001 \\
\hline & & & & & \\
Foodstuffs & 7.40 & 126.6 & 127 & 135.3 & 136.6 \\
Other food products & 11.01 & 121.6 & 125 & 131.1 & 135.2 \\
Beverages and tobacco & 6.49 & 117.1 & 116.3 & 120.8 & 134.9 \\
Textiles & 11.33 & 97.3 & 98.4 & 102 & 101.9 \\
Clothing (excluding shoes) & 9.04 & 147.5 & 142.1 & 138.7 & 131.2 \\
Leather and shoes & 2.04 & 114.1 & 111.2 & 114.8 & 120.9 \\
Wood and wood products & 2.07 & 105.4 & 106.2 & 110.8 & 116.8 \\
Paper products & 3.69 & 128.4 & 135.1 & 148.2 & 153 \\
Processed mineral ores & 10.82 & 114.0 & 117.3 & 121.3 & 125.4 \\
Metallurgical products & 2.19 & 121.0 & 135.0 & 135.3 & 144.8 \\
Metal products & 6.08 & 100.5 & 105.4 & 109 & 118.8 \\
Machinery (excluding transport) & 1.94 & 103.4 & 109.2 & 120.5 & 118.2 \\
Transport equipment & 4.06 & 116.6 & 120.3 & 125.5 & 143.4 \\
Electronic equipment & 3.42 & 115.8 & 126.4 & 127.1 & 127.4 \\
Office, measuring, optical equipment & 0.21 & 102.0 & 136.9 & 159.6 & 114.9 \\
Chemicals & 15.09 & 120.0 & 125.7 & 130 & 137.7 \\
Rubber and plastic products & 2.97 & 122.2 & 127.7 & 126.3 & 122.9 \\
Other manufacturing & 0.14 & 109.7 & 108.6 & 111.7 & 108.8 \\
& & & & & \\
Total manufacturing industry & 100.00 & 117.9 & 120.7 & 124.9 & 128.9 \\
& & & & & \\
\hline
\end{tabular}

Source: Moroccan authorities. 
Table 7. Morocco: Foreign Direct Investment by Sector, 1998-2002 1/ (In millions of Moroccan dirhams)

\begin{tabular}{lrrrrr}
\hline & 1998 & 1999 & 2000 & 2001 & $\frac{\text { Prel. }}{2002}$ \\
\hline Real estate & 533 & 488 & 655 & 865 & 1,048 \\
& & & & & \\
Industry 2/ & 2,046 & 3,783 & 1,152 & 2,510 & 1,189 \\
Fishing & 44 & 4 & 14 & 71 & 18 \\
Tourism & 167 & 304 & 121 & 191 & 344 \\
Services & 236 & 359 & 304 & 991 & 817 \\
Transport & 9 & 34 & 8 & 28 & 2 \\
Public works & 28 & 130 & 85 & 123 & 5 \\
Banking & 1,159 & 2,154 & 758 & 358 & 218 \\
Telecommunication & 13 & 10,175 & 7,981 & 26,377 & 328 \\
Other & 1,198 & 1,029 & 1,481 & 1,592 & 606 \\
Total & 5,433 & 18,460 & 12,639 & 33,260 & 4,324 \\
$\quad$ Of which: & & & & & \\
$\quad$ Foreign exchange & 4,472 & 17,197 & 12,444 & 32,474 & 4,058 \\
$\quad$ Other & 961 & 1,263 & 5,195 & 786 & 266 \\
\hline
\end{tabular}

Source: Moroccan authorities.

1/ Including loans provided by foreign companies to their subsidiaries in Morocco.

2/ Including textiles. 
Table 8. Morocco: Consumer Price, Wholesale Price, and Producer Price Indices, 1998--2001

(Annual percentage change)

\begin{tabular}{|c|c|c|c|c|}
\hline & 1998 & 1999 & 2000 & 2001 \\
\hline \multicolumn{5}{|l|}{ Consumer price index $1 /$} \\
\hline Food & 3.1 & -0.8 & 1.5 & -1.0 \\
\hline Clothing & 3.8 & 3.1 & 2.4 & 1.4 \\
\hline Housing & 2.5 & 2.8 & 1.7 & 2.1 \\
\hline Household goods & 1.8 & 1.4 & 0.2 & 0.1 \\
\hline Medical services & 3.3 & 1.2 & 0.5 & 1.4 \\
\hline Transport and communications & 2.8 & 1.7 & 5.2 & 2.6 \\
\hline Entertainment and culture & 1.8 & 1.3 & 2.2 & 3.6 \\
\hline Other goods and services & 1.7 & 1.5 & 2.1 & 2.1 \\
\hline General consumer price index & 2.7 & 0.7 & 1.9 & 0.6 \\
\hline \multicolumn{5}{|l|}{ Wholesale price index $2 /$} \\
\hline Agricultural sector & 2.5 & -1.3 & 3.1 & -0.3 \\
\hline Cereals & 4.2 & -3.1 & 2.8 & 3.0 \\
\hline Industrial crops & 2.7 & -0.3 & -2.4 & 2.2 \\
\hline Vegetables & 14.0 & -13.2 & 18.2 & -12.2 \\
\hline Fruits & 5.8 & 5.5 & 6.4 & 12.1 \\
\hline Animal products & -3.5 & -0.7 & -4.8 & -3.9 \\
\hline Forestry products & 3.2 & 10.0 & 35.0 & -5.1 \\
\hline Fishing & 11.3 & -11.9 & -1.4 & -6.7 \\
\hline \multicolumn{5}{|l|}{ Price index of industrial production } \\
\hline Mining & 7.1 & -1.0 & 6.9 & -1.0 \\
\hline Electricity and water & 4.7 & 6.7 & -1.9 & -5.8 \\
\hline Manufacturing industries & -2.6 & 0.5 & 9.3 & -1.8 \\
\hline Foodstuffs & 1.0 & -1.1 & -0.1 & -0.6 \\
\hline Textiles & 0.3 & 0.6 & -3.8 & 0.3 \\
\hline Metallurgical industries & -0.3 & -1.9 & 3.4 & -1.3 \\
\hline
\end{tabular}

Source: Ministry of Economic Forecasting and Planning,Direction de la Statistique.

1/ Consumer price index based on price movements of 385 articles in the cities of Agad: Casablanca, Fes, Kenitra, Laayoune, Marrakesh, Meknes, Oujda, Rabat, Tangier, and ' $2 /$ Index based on price movements of 70 articles. 
Table 9. Morocco: Evolution of Consumer Subsidies and Retail Prices, 1998/99-2002 1/

\begin{tabular}{|c|c|c|c|c|c|c|}
\hline & Units & $1998 / 99$ & $1999 / 00$ & $20002 /$ & 2001 & $\frac{\text { Prel. }}{2002}$ \\
\hline \multicolumn{7}{|l|}{ Sugar } \\
\hline Quantities subsidized & $\mathrm{Mil} / \mathrm{kos}$ & 924 & 969 & 462 & 997 & 1,015 \\
\hline Cubes & & 478 & 503 & 237 & 518 & 528 \\
\hline Granulated & & 446 & 466 & 225 & 479 & 487 \\
\hline Cost per unit 3/ & $\mathrm{DH} / \mathrm{kos}$ & & & & & \\
\hline Cubes & & 6.95 & 6.95 & 6.95 & 6.95 & 6.95 \\
\hline Granulated & & 6.00 & 6.00 & 6.00 & 6.00 & 6.00 \\
\hline Retail price & $\mathrm{DH} / \mathrm{kos}$ & & & & & \\
\hline Cubes & & 4.95 & 4.95 & 4.95 & 4.95 & 4.95 \\
\hline Granulated & & 4.00 & 4.00 & 4.00 & 4.00 & 4.00 \\
\hline Subsidy per unit & $\mathrm{DH} / \mathrm{kos}$ & & & & & \\
\hline Cubes & & 2.00 & 2.00 & 2.00 & 2.00 & 2.00 \\
\hline Granulated & & 2.00 & 2.00 & 2.00 & 2.00 & 2.00 \\
\hline Subsidy/cost per unit & Percent & & & & & \\
\hline Cubes & & 29 & 29 & 29 & 29 & 29 \\
\hline Granulated & & 33 & 33 & 33 & 33 & 33 \\
\hline Amount of subsidies & $\mathrm{Mil} / \mathrm{DH}$ & 1,849 & 1,938 & 924 & 1,994 & 2,030 \\
\hline \multicolumn{7}{|l|}{ Edible oils } \\
\hline Quantities subsidized & Mil/liters & 380 & 410 & 187 & n.a. & n.a. \\
\hline Cost per unit & DH/liters & 13.28 & 13.28 & 13.28 & n.a. & n.a. \\
\hline Average selling price & DH/liters & 8.40 & 8.40 & 8.40 & n.a. & n.a. \\
\hline Subsidy per unit & $\mathrm{DH} /$ liters & 4.88 & 4.88 & 4.88 & n.a. & n.a. \\
\hline Subsidy/cost per unit & Percent & 37 & 37 & 37 & n.a. & n.a. \\
\hline Amount of subsidies & $\mathrm{Mil} / \mathrm{DH}$ & 1,852 & 2,001 & 912 & 62 & 28 \\
\hline \multicolumn{7}{|l|}{ Flour } \\
\hline Quantities subsidized & Mil/kos & 1,000 & 1,000 & 490 & 1,000 & 1,000 \\
\hline Cost per unit & $\mathrm{DH} / \mathrm{kos}$ & 3.92 & 3.92 & 3.92 & 3.92 & 3.98 \\
\hline Retail price & $\mathrm{DH} / \mathrm{kos}$ & 2.00 & 2.00 & 2.00 & 2.00 & 2.00 \\
\hline Subsidy per unit & $\mathrm{DH} / \mathrm{kos}$ & 1.92 & 1.92 & 1.92 & 1.92 & 1.92 \\
\hline Subsidy/cost per unit & Percent & 49 & 49 & 49 & 49 & 50 \\
\hline Amount of subsidies & $\mathrm{Mil} / \mathrm{DH}$ & 1,901 & 1,920 & 940 & 1,923 & 1,980 \\
\hline Petroleum products & $\mathrm{Mil} / \mathrm{DH}$ & $\ldots$ & 400 & 800 & 3,144 & 0 \\
\hline Total subsidies & $\mathrm{Mil} / \mathrm{DH}$ & 5,602 & 6,259 & 3,576 & 7,123 & 4,038 \\
\hline
\end{tabular}

Source: Ministry of Finance, Direction des établissements publics et des participations.

1/ Based on July-June fiscal year basis in 1998/99 and 1999/2000; calendar year from 2001.

2/ Second semester.

3/ Sum of retail prices and subsidies per unit. 
Table 10. Morocco: National Minimum Wages, July 1996-January 2003

\begin{tabular}{lrr}
\hline & $\begin{array}{r}\text { July 1996- } \\
\text { June 2000 }\end{array}$ & $\begin{array}{r}\text { July 2000- } \\
\text { January 2003 }\end{array}$ \\
\hline SMIG (dirhams per hour) 1/ & 7.98 & 8.78 \\
SMAG (dirhams per day) 2/ & 41.36 & 45.5 \\
\hline
\end{tabular}

Source: Moroccan authorities.

1/ Salaire minimum interprofessionnel garanti.

2/ Salaire minimum agricole garanti. 
Table 11. Morocco. Urban Population by Activity, 1998-2002 1/

\begin{tabular}{|c|c|c|c|c|c|c|c|c|c|c|c|c|c|c|c|}
\hline & \multicolumn{3}{|c|}{1998} & \multicolumn{3}{|c|}{1999} & \multicolumn{3}{|c|}{2000} & \multicolumn{3}{|c|}{2001} & \multicolumn{2}{|r|}{2002} & \multirow{2}{*}{$\frac{\text { Prel. }}{\text { Total }}$} \\
\hline & Men & Women & Total & Men & Women & Total & Men & Women & Total & Men & Women & Total & Men & Women & \\
\hline & \multicolumn{15}{|c|}{ (In thousands; unless otherwise specified) } \\
\hline Total population & 13,819 & 13,956 & 27,775 & 14,049 & 14,189 & 28,238 & 14,281 & 14,424 & 28,706 & 14512 & 14659 & 29171 & 14694 & 14841 & 29535 \\
\hline Total urban population & 7,372 & 7,584 & 14,956 & 7,580 & 7,821 & 15,401 & 7,787 & 8,062 & 15,849 & 8000 & 8308 & 16308 & 8172 & 8504 & 16676 \\
\hline In percent of total & 53.3 & 54.3 & 53.8 & 54.0 & 55.1 & 54.5 & 54.5 & 55.9 & 55.2 & 55.1 & 56.7 & 55.9 & 55.6 & 57.3 & 56.5 \\
\hline Unemployed $2 /$ & 676 & 293 & 969 & 808 & 354 & 1,162 & 814 & 333 & 1,146 & 760 & 301 & 1061 & 716 & 294 & 1010 \\
\hline Inactive & 3,457 & 6,361 & 9,818 & 3,545 & 6,519 & 10,064 & 3,649 & 6,799 & 10,448 & 3756 & 7072 & 10828 & 3865 & 7314 & 11179 \\
\hline Employed 3/ & 3,238 & 930 & 4,168 & 3,226 & 948 & 4,174 & 3,324 & 930 & 4,255 & 3485 & 934 & 4419 & 3613 & 930 & 4543 \\
\hline Total uban active population 4/ & 3,914 & 1,223 & 5,137 & 4,034 & 1,302 & 5,336 & 4,138 & 1,263 & 5,401 & 4245 & 1235 & 5480 & 4307 & 1190 & 5497 \\
\hline Rate of activity (percent) & 53.1 & 16.1 & 34.4 & 53.2 & 16.6 & 34.6 & 53.1 & 15.7 & 34.1 & 53.1 & 14.9 & 33.6 & 52.7 & 14 & 33 \\
\hline Rate of umemployment (percent) & 17.5 & 24.4 & 19.1 & 20.3 & 27.6 & 22.0 & 19.9 & 26.7 & 21.5 & 18 & 24.7 & 19.5 & 16.2 & 24.3 & 18 \\
\hline \multicolumn{16}{|c|}{ Rate of unemployment by age (percent) } \\
\hline $15-24$ years & 34.7 & 35.9 & 35.0 & 37.8 & 37.9 & 37.8 & 37.8 & 37.0 & 37.6 & 35.4 & 35.8 & 35.5 & 32.7 & 36.6 & 33.7 \\
\hline $25-59$ years & 13.6 & 20.6 & 15.2 & 16.4 & 24.5 & 18.3 & 16.1 & 24.0 & 17.9 & 14.6 & 21.9 & 16.2 & 13.2 & 21.3 & 15 \\
\hline 60 years and over & 1.2 & 2.3 & 1.4 & 1.9 & 1.1 & 1.8 & 1.3 & 2.1 & 1.4 & 0.8 & 2.5 & 1 & 0.5 & 0.4 & 0.5 \\
\hline
\end{tabular}

Source: Ministry of Population.

1/ Data are based on annual population and employment surveys.

2/ Persons over 15 years old seeking work.

3/ Employed persons over 7 years old.

4/ Employed and unemployed. 
Table 12. Morocco: Financial Transactions of the Central Government, 1998/99-2002

\begin{tabular}{|c|c|c|c|c|c|c|}
\hline & \multicolumn{5}{|c|}{ 2nd half } & \multirow{2}{*}{$\begin{array}{l}\text { Prel. } \\
2002\end{array}$} \\
\hline & $1998 / 99$ & $1999 / 00$ & $20001 /$ & 2000 & 2001 & \\
\hline & \multicolumn{6}{|c|}{ (In millions of Moroccan dirhams) } \\
\hline Total revenue (excluding privatization) & 93,809 & 94,168 & 43,324 & 92,858 & 95,384 & 98,261 \\
\hline Taxes on income and profits & 25,010 & 26,938 & 12,067 & 26,841 & 28,162 & 30,378 \\
\hline Corporate profit tax (IS) & 9,512 & 10,299 & 4,317 & 10,124 & 11,708 & 12,917 \\
\hline Income tax (IGR) & 10,824 & 11,803 & 5,505 & 11,967 & 15,338 & 16,353 \\
\hline Other & 4,674 & 4,836 & 2,245 & 4,750 & 1,116 & 1,108 \\
\hline Taxes on international trade & 16,299 & 16,896 & 7,895 & 16,636 & 14,010 & 14,231 \\
\hline Domestic taxes on goods and services & 34,412 & 36,999 & 18,697 & 36,659 & 38,994 & 40,056 \\
\hline VAT & 19,136 & 21,157 & 11,027 & 21,476 & 23,115 & 23,951 \\
\hline Excise taxes & 15,276 & 15,842 & 7,670 & 15,183 & 15,879 & 16,105 \\
\hline Road Fund revenues (Fonds Routier) & 1,130 & 1,233 & 515 & 1,175 & 1,259 & 1,356 \\
\hline Registration and stamp taxes & 3,892 & 4,130 & 1,686 & 4,162 & 4,546 & 4,999 \\
\hline Tax amnesty revenues & 3,623 & 0 & 0 & 0 & 0 & 0 \\
\hline Dividend and license income & 5,759 & 5,683 & 1,352 & 5,287 & 4,904 & 4,244 \\
\hline Other nontax revenue & 3,684 & 2,289 & 1,112 & 2,098 & 3,509 & 2,997 \\
\hline Total expenditure & 102,638 & 109,767 & 60,725 & 114,626 & 119,850 & 118,999 \\
\hline Current expenditure & 80,664 & 86,459 & 43,869 & 87,294 & 90,412 & 90,691 \\
\hline Goods and services & 56,789 & 59,405 & 29,936 & 59,296 & 66,534 & 69,033 \\
\hline Wages and salaries $2 /$ & 40,286 & 42,132 & 21,222 & 41,967 & 47,998 & 48,580 \\
\hline Other & 16,503 & 17,273 & 8,714 & 17,329 & 18,536 & 20,453 \\
\hline Interest payments & 17,731 & 18,865 & 9,643 & 18,620 & 18,754 & 17,611 \\
\hline Domestic & 11,088 & 12,670 & 6,250 & 12,317 & 12,846 & 13,174 \\
\hline External & 6,643 & 6,195 & 3,393 & 6,303 & 5,908 & 4,437 \\
\hline Food and petroleum subsidies $3 /$ & 6,144 & 8,189 & 4,290 & 9,378 & 5,124 & 4,047 \\
\hline Transfers to local governments & 5,741 & 6,347 & 3,308 & 6,443 & 6,935 & 7,185 \\
\hline Capital expenditure & 17,428 & 17,485 & 13,648 & 21,151 & 21,866 & 21,123 \\
\hline Net lending & $-1,195$ & -524 & -100 & -262 & 637 & -300 \\
\hline Balance of Special Treasury Accounts & 376 & 777 & 700 & $-1,039$ & 2,100 & 2,117 \\
\hline Overall balance & $-8,453$ & $-14,822$ & $-16,701$ & $-22,807$ & $-22,366$ & $-18,621$ \\
\hline Pivatization receipts & 392 & 11,131 & 18 & 18 & 23,372 & 621 \\
\hline Variation in arrears ( $+=$ accumulation) & $-5,029$ & 1,537 & 11,915 & 13,809 & $-10,996$ & -231 \\
\hline Financing requirement & $-13,090$ & $-2,154$ & $-4,768$ & $-8,980$ & $-9,990$ & $-18,231$ \\
\hline Financing & 13,090 & 2,154 & 4,768 & 8,980 & 9,990 & 18,231 \\
\hline Domestic & 18,281 & 9,179 & 7,004 & 15,299 & 19,788 & 29,430 \\
\hline Banking sector $4 /$ & $-1,384$ & $-3,419$ & 6,751 & 9,719 & $-6,885$ & 4,163 \\
\hline Nonbanking sector & 19,665 & 12,598 & 129 & 5,580 & 26,673 & 25,267 \\
\hline Extemal & $-5,191$ & $-7,025$ & $-2,236$ & $-6,319$ & $-9,798$ & $-11,199$ \\
\hline Net drawings & $-5,191$ & $-7,536$ & $-2,236$ & $-6,319$ & $-10,053$ & $-11,699$ \\
\hline Drawings & 8,665 & 4,816 & 3,810 & 5,729 & 3,150 & 3,984 \\
\hline \multirow[t]{2}{*}{ Amortization } & $-13,856$ & $-12,352$ & $-6,046$ & $-12,048$ & $-13,203$ & $-15,683$ \\
\hline & \multicolumn{6}{|c|}{ (In percent of GDP) } \\
\hline Total revenue (excluding privatization) & 27.2 & 27.2 & 24.8 & 26.6 & 24.9 & 24.0 \\
\hline Total expenditure & 29.8 & 31.7 & 34.7 & 32.8 & 31.3 & 29.0 \\
\hline Current (including net lending) & 23.0 & 24.8 & 25.0 & 24.9 & 23.8 & 22.1 \\
\hline Capital (including tranfers to local governments) & 6.7 & 6.9 & 9.7 & 7.9 & 7.5 & 6.9 \\
\hline Overall balance, payment-order basis & & & & & & \\
\hline excluding privatization receipts & -2.5 & -4.3 & -9.6 & -6.5 & -5.8 & -4.5 \\
\hline Privatization revenue receipts & 0.1 & 3.2 & 0.0 & 0.0 & 6.1 & 0.2 \\
\hline Variation in arrears (+=accumulation) & -1.5 & 0.4 & 6.8 & 3.9 & -2.9 & -0.1 \\
\hline Financing requirement & -3.8 & -0.6 & -2.7 & -2.6 & -2.6 & -4.5 \\
\hline
\end{tabular}

Sources: Ministry of Finance; and Bank Al-Maghrib.

1/ Transition fiscal year July-December 2000.

$2 /$ Includes wages of security and military personnel.

3/ Petroleum product subsidies (DM 3.4 billion) are imputed to the budget year in which the arrears were incurred, as estimated on the basis of the difference between domestic and international petroleum prices.

4/ Differs from monetary data owing to differences in coverage. 
Table 13. Morocco: Central Government Operations, 1998/99-2002

(Fiscal years; in millions of Moroccan dirhams)

\begin{tabular}{|c|c|c|c|c|c|c|c|}
\hline & $1998 / 99$ & $\begin{array}{l}\text { Budget } \\
999 / 2000\end{array}$ & $999 / 2000$ & $\begin{array}{l}\text { 2nd half } \\
20001 /\end{array}$ & 2000 & 2001 & $\begin{array}{l}\text { Prel. } \\
2002\end{array}$ \\
\hline $\begin{array}{l}\text { Total revenue, excluding } \\
\text { receipts from privatization }\end{array}$ & 93,809 & 86,659 & 94,168 & 43,324 & 92,858 & 95,384 & 98,261 \\
\hline Tax revenue & 84,366 & 78,516 & 86,196 & 40,860 & 85,473 & 86,971 & 91,020 \\
\hline Taxes on income and profits & 25,010 & 23,454 & 26,938 & 12,067 & 26,841 & 28,162 & 30,378 \\
\hline Licences & 299 & 299 & 288 & 100 & 287 & 304 & 339 \\
\hline Corporate (IS and IBP, excl. OCP) & 9,512 & 8,026 & 10,299 & 4,317 & 10,124 & 11,708 & 12,917 \\
\hline Individual (IGR) & 10,824 & 10,775 & 11,803 & 5,505 & 11,967 & 15,338 & 16,353 \\
\hline Dividend income (TPA) & 478 & 478 & 406 & 269 & 408 & 0 & 0 \\
\hline Real estate capital gains (TPI) & 543 & 543 & 490 & 294 & 527 & 0 & 0 \\
\hline Solidarity (PSN) & 509 & 509 & 373 & 101 & 367 & 285 & 152 \\
\hline Interest income (TRPRF) & 2,397 & 2,397 & 2,474 & 1,235 & 2,452 & 0 & 0 \\
\hline Penalties and fines & 258 & 258 & 351 & 161 & 339 & 399 & 478 \\
\hline Other & 190 & 190 & 454 & 85 & 370 & 128 & 139 \\
\hline Taxes on international trade & 16,299 & 15,783 & 16,896 & 7,895 & 16,636 & 14,010 & 14,231 \\
\hline Customs duties & 6,661 & 6,631 & 6,617 & 6,357 & 12,810 & 12,350 & 12,233 \\
\hline Fiscal tax (PFI) & 6,046 & 6,019 & 6,097 & 0 & 0 & 0 & 0 \\
\hline Tariff surcharges $2 /$ & 3,555 & 3,096 & 4,150 & 1,538 & 3,826 & 1,660 & 1,998 \\
\hline Other taxes & 37 & 37 & 32 & 0 & 0 & 0 & 0 \\
\hline Taxes on goods and services & 34,412 & 34,257 & 36,999 & 18,697 & 36,659 & 38,994 & 40,056 \\
\hline VAT & 19,136 & 19,737 & 21,157 & 11,027 & 21,476 & 23,115 & 23,951 \\
\hline Domestic & 8,253 & 0 & 9,529 & 4,734 & 9,213 & 10,482 & 10,751 \\
\hline Imports & 10,883 & 0 & 11,629 & 6,293 & 12,263 & 12,633 & 13,200 \\
\hline Excises (TIC) & 15,276 & 15,276 & 15,842 & 7,670 & 15,183 & 15,879 & 16,105 \\
\hline Tobacco products & 5,284 & 0 & 5,438 & 2,819 & 5,442 & 5,627 & 5,801 \\
\hline Petroleum products & 9,028 & 0 & 9,450 & 4,420 & 8,795 & 9,239 & 9,308 \\
\hline Other & 964 & 0 & 954 & 431 & 946 & 1,013 & 996 \\
\hline Revenue accruing to the Road Fund & 1,130 & 1,130 & 1,233 & 515 & 1,175 & 1,259 & 1,356 \\
\hline Registration and stamp taxes & 3,892 & 3,892 & 4,130 & 1,686 & 4,162 & 4,546 & 4,999 \\
\hline Tax amnesty payments & 3,623 & 0 & 0 & 0 & 0 & 0 & 0 \\
\hline Nontax revenue & 9,443 & 8,143 & 7,972 & 2,464 & 7,385 & 8,413 & 7,241 \\
\hline Dividend and license income & 5,759 & 4,459 & 5,683 & 1,352 & 5,287 & 4,904 & 4,244 \\
\hline Property income & 137 & 137 & 134 & 62 & 121 & 168 & 141 \\
\hline Other revenue & 3,547 & 3,547 & 2,155 & 1,050 & 1,977 & 3,341 & 2,856 \\
\hline Total expenditure & 102,638 & 102,219 & 109,767 & 60,725 & 114,626 & 119,850 & 118,699 \\
\hline
\end{tabular}


Table 13. Morocco: Central Government Operations, 1998/99-2002 (concluded)

(Fiscal years; in millions of Moroccan dirhams)

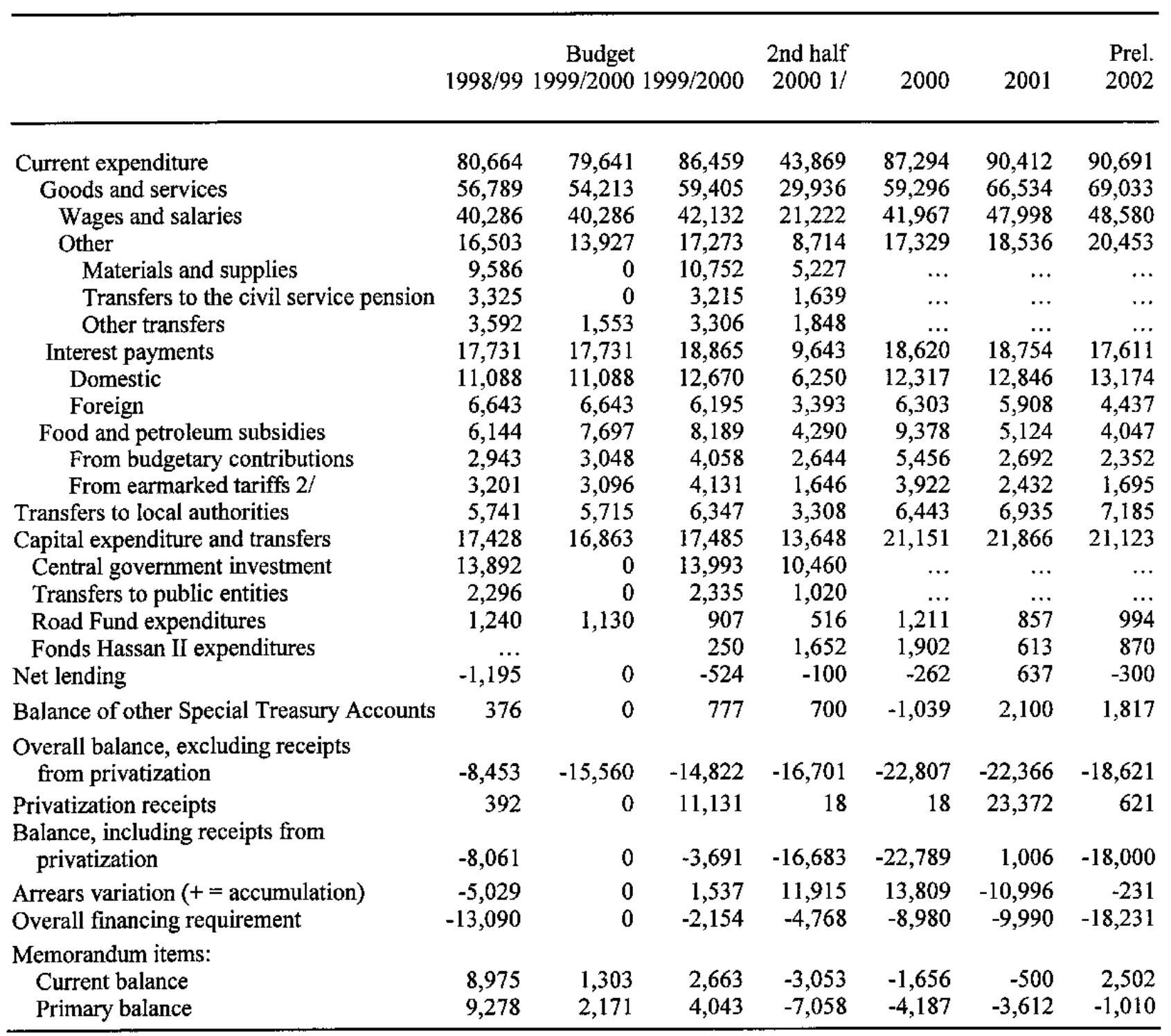

Source: Ministry of Finance.

1/ Transition fiscal year July-December 2000.

2/ Equivalents tarifaires. 
Table 14. Morocco: Central Government Revenue by Main Categories, 1998/99-2002

\begin{tabular}{|c|c|c|c|c|c|c|}
\hline & \multicolumn{5}{|c|}{ 2nd half } & \multirow{2}{*}{$\frac{\text { Prel. }}{2002}$} \\
\hline & $1998 / 99$ & $1999 / 00$ & $20001 /$ & 2000 & 2001 & \\
\hline & \multicolumn{6}{|c|}{ (In millions of Moroccan dirhams) } \\
\hline Total revenue $2 /$ & 93,809 & 94,168 & 43,324 & 92,858 & 95,384 & 98,261 \\
\hline Tax revenue & 84,366 & 86,196 & 40,860 & 85,473 & 86,971 & 91,020 \\
\hline Corporate taxes & 9,512 & 10,299 & 4,317 & 10,124 & 11,708 & 12,917 \\
\hline Individual income tax & 10,824 & 11,803 & 5,505 & 11,967 & 15,338 & 16,353 \\
\hline Custom duties and taxes & 16,299 & 16,896 & 7,895 & 16,636 & 14,010 & 14,231 \\
\hline VAT & 19,136 & 21,157 & 11,027 & 21,476 & 23,115 & 23,951 \\
\hline domestic & 8,253 & 9,529 & 4,734 & 9,213 & 10,482 & 10,751 \\
\hline imports & 10,883 & 11,629 & 6,293 & 12,263 & 12,633 & 13,200 \\
\hline Excises & 15,276 & 15,842 & 7,670 & 15,183 & 15,879 & 16,105 \\
\hline Registration and stamp taxes & 3,892 & 4,130 & 1,686 & 4,162 & 4,546 & 4,999 \\
\hline Other taxes & 9,427 & 6,069 & 2,760 & 5,925 & 2,375 & 2,464 \\
\hline \multirow[t]{2}{*}{ Nontax revenue } & 9,443 & 7,972 & 2,464 & 7,385 & 8,413 & 7,241 \\
\hline & \multicolumn{6}{|c|}{ (In percent of GDP) } \\
\hline Total revenue $2 /$ & 27.2 & 27.2 & 24.8 & 26.6 & 24.9 & 24.0 \\
\hline Tax revenue & 24.5 & 24.9 & 23.4 & 24.4 & 22.7 & 22.2 \\
\hline Corporate taxes & 2.8 & 3.0 & 2.5 & 2.9 & 3.1 & 3.2 \\
\hline Individual income tax & 3.1 & 3.4 & 3.1 & 3.4 & 4.0 & 4.0 \\
\hline Import duties and taxes & 4.7 & 4.9 & 4.5 & 4.8 & 3.7 & 3.5 \\
\hline VAT & 5.5 & 6.1 & 6.3 & 6.1 & 6.0 & 5.8 \\
\hline domestic & 2.4 & 2.8 & 2.7 & 2.6 & 2.7 & 2.6 \\
\hline imports & 3.2 & 3.4 & 3.6 & 3.5 & 3.3 & 3.2 \\
\hline Excises & 4.4 & 4.6 & 4.4 & 4.3 & 4.1 & 3.9 \\
\hline Registration and stamp taxes & 1.1 & 1.2 & 1.0 & 1.2 & 1.2 & 1.2 \\
\hline Other taxes & 2.7 & 1.8 & 1.6 & 1.7 & 0.6 & 0.6 \\
\hline \multirow[t]{2}{*}{ Nontax revenue } & 2.7 & 2.3 & 1.4 & 2.1 & 2.2 & 1.8 \\
\hline & \multicolumn{6}{|c|}{ (In percent of total revenue) } \\
\hline Total revenue $2 /$ & 100.0 & 100.0 & 100.0 & 100.0 & 100.0 & 100.0 \\
\hline Tax revenue & 89.9 & 91.5 & 94.3 & 92.0 & 91.2 & 92.6 \\
\hline Corporate taxes & 10.1 & 10.9 & 10.0 & 10.9 & 12.3 & 13.1 \\
\hline Individual income tax & 11.5 & 12.5 & 12.7 & 12.9 & 16.1 & 16.6 \\
\hline Import duties and taxes & 17.4 & 17.9 & 18.2 & 17.9 & 14.7 & 14.5 \\
\hline Export taxes & 0.0 & 0.0 & 0.0 & 0.0 & 0.0 & 0.0 \\
\hline VAT & 20.4 & 22.5 & 25.5 & 23.1 & 24.2 & 24.4 \\
\hline domestic & 8.8 & 10.1 & 10.9 & 9.9 & 11.0 & 10.9 \\
\hline imports & 11.6 & 12.3 & 14.5 & 13.2 & 13.2 & 13.4 \\
\hline Excises & 16.3 & 16.8 & 17.7 & 16.4 & 16.6 & 16.4 \\
\hline Registration and stamp taxes & 4.1 & 4.4 & 3.9 & 4.5 & 4.8 & 5.1 \\
\hline Other taxes & 10.0 & 6.4 & 6.4 & 6.4 & 2.5 & 2.5 \\
\hline Nontax revenue & 10.1 & 8.5 & 5.7 & 8.0 & 8.8 & 7.4 \\
\hline
\end{tabular}

Source: Ministry of Finance.

1/ Transition fiscal year July-December 2000.

2/ Excludes revenue from privatization. 
Table 15. Morocco: Economic Classification of Central Government Expenditure, 1998/99-2002

\begin{tabular}{|c|c|c|c|c|c|c|}
\hline \multirow[b]{4}{*}{ Total expenditure (excl. net lending) } & \multicolumn{5}{|c|}{ 2nd half } & \multirow{2}{*}{$\underline{\text { Prel }}$} \\
\hline & $1998 / 99$ & $1999 / 2000$ & $20001 /$ & 2000 & 2001 & \\
\hline & \multicolumn{6}{|c|}{ (In millions of Moroccan dirhams) } \\
\hline & $103,832.8$ & 110,291 & 60,825 & 114,888 & 119,213 & 118,999 \\
\hline Current expenditure & $80,664.0$ & 86,459 & 43,869 & 87,294 & 90,412 & 90,691 \\
\hline Goods and services & $56,789.0$ & 59,405 & 29,936 & 59,296 & 66,534 & 69,033 \\
\hline Wages and salaries & $40,286.0$ & 42,132 & 21,222 & 41,967 & 47,998 & 48,580 \\
\hline Other & $16,503.0$ & 17,273 & 8,714 & 17,329 & 18,536 & 20,453 \\
\hline Materials and supplies & $9,586.0$ & 10,752 & 5,227 & $\ldots$ & $\ldots$ & $\ldots$ \\
\hline Civil service pension fund & $3,325.0$ & 3,215 & 1,639 & $\ldots$ & $\ldots$ & $\ldots$ \\
\hline Other transfers & $3,592.0$ & 3,306 & 1,848 & $\ldots$ & $\ldots$ & $\ldots$ \\
\hline Interest payments & $17,731.0$ & 18,865 & 9,643 & 18,620 & 18,754 & 17,611 \\
\hline Domestic & $11,088.0$ & 12,670 & 6,250 & 12,317 & 12,846 & 13,174 \\
\hline Foreign & $6,643.0$ & 6,195 & 3,393 & 6,303 & 5,908 & 4,437 \\
\hline Food subsidies & $6,144.0$ & 8,189 & 4,290 & 9,378 & 5,124 & 4,047 \\
\hline From budgetary contribution & $2,943.0$ & 4,058 & 2,644 & 5,456 & 2,692 & 2,352 \\
\hline From earmarked tariffs & $3,201.0$ & 4,131 & 1,646 & 3,922 & 2,432 & 1,695 \\
\hline Transfers to local governments & $5,740.8$ & 6,347 & 3,308 & 6,443 & 6,935 & 7,185 \\
\hline Capital expenditure and transfers & $17,428.0$ & 17,485 & 13,648 & 21,151 & 21,866 & 21,123 \\
\hline Central government investment & $13,891.8$ & 13,993 & 10,460 & $\ldots$ & $\ldots$ & $\ldots$ \\
\hline Capital transfers to public enterprises & $2,296.2$ & 2,335 & 1,020 & $\ldots$ & $\ldots$ & $\ldots$ \\
\hline Fonds Hassan II expenditures & & 250 & 1,652 & 1,902 & 613 & 870 \\
\hline \multirow[t]{2}{*}{ Road Fund expenditures } & $1,240.0$ & 907 & 516 & 1,211 & 857 & 994 \\
\hline & \multicolumn{6}{|c|}{ (In percent of GDP) } \\
\hline Total expenditure (excl. net lending) & 30.1 & 31.8 & 17.4 & 32.9 & 31.1 & 29.1 \\
\hline Current expenditure & 23.4 & 25.0 & 12.5 & 25.0 & 23.6 & 22.2 \\
\hline Goods and services & 16.5 & 17.1 & 8.6 & 17.0 & 17.4 & 16.9 \\
\hline Wages and salaries & 11.7 & 12.2 & 6.1 & 12.0 & 12.5 & 11.9 \\
\hline Other & 4.8 & 5.0 & 2.5 & 5.0 & 4.8 & 5.0 \\
\hline Materials and supplies & 2.8 & 3.1 & 1.5 & $\cdots$ & $\ldots$ & $\ldots$ \\
\hline Transfers to the civil service pension fund & 1.0 & 0.9 & 0.5 & $\cdots$ & $\cdots$ & $\cdots$ \\
\hline Other transfers & 1.0 & 1.0 & 0.5 & $\ldots$ & $\ldots$ & $\ldots$ \\
\hline Interest payments & 5.1 & 5.4 & 2.8 & 5.3 & 4.9 & 4.3 \\
\hline Domestic & 3.2 & 3.7 & 1.8 & 3.5 & 3.4 & 3.2 \\
\hline Foreign & 1.9 & 1.8 & 1.0 & 1.8 & 1.5 & 1.1 \\
\hline Food subsidies & 1.8 & 2.4 & 1.2 & 2.7 & 1.3 & 1.0 \\
\hline From budgetary contribution & 1.8 & 2.4 & 1.2 & 2.7 & 1.3 & 1.0 \\
\hline From earmarked tariffs & 0.9 & 1.2 & 0.5 & 1.1 & 0.6 & 0.4 \\
\hline Transfers to local governments & 1.7 & 1.8 & 0.9 & 1.8 & 1.8 & 1.8 \\
\hline Capital expenditure and transfers & 5.1 & 5.0 & 3.9 & 6.0 & 5.7 & 5.2 \\
\hline Central government investment & 4.0 & 4.0 & 3.0 & $\ldots$ & $\ldots$ & $\ldots$ \\
\hline Capital transfers to public enterprises & 0.7 & 0.7 & 0.3 & $\ldots$ & $\ldots$ & $\ldots$ \\
\hline Fonds Hassan II expenditures & $\ldots$ & $\ldots$ & $\ldots$ & $\ldots$ & $\ldots$ & $\ldots$ \\
\hline Road Fund expenditures & 0.4 & 0.3 & 0.1 & 0.3 & 0.2 & 0.2 \\
\hline
\end{tabular}


Table 15. Morocco: Economic Classification of Central Government Expenditure, 1998/99-2002 (concluded)

\begin{tabular}{|c|c|c|c|c|c|c|}
\hline & $1998 / 99$ & $1999 / 2000$ & $\begin{array}{l}\text { 2nd half } \\
20001 /\end{array}$ & 2000 & 2001 & $\frac{\text { Prel }}{2002}$ \\
\hline & \multicolumn{6}{|c|}{ (In percent of total expenditure) } \\
\hline Total expenditure (excl. net lending) & 100.0 & 100.0 & 100.0 & 100.0 & 100.0 & 100.0 \\
\hline Current expenditure & 77.7 & 78.4 & 72.1 & 76.0 & 75.8 & 76.2 \\
\hline Goods and services & 54.7 & 53.9 & 49.2 & 51.6 & 55.8 & 58.0 \\
\hline Wages and salaries & 38.8 & 38.2 & 34.9 & 36.5 & 40.3 & 40.8 \\
\hline Other & 15.9 & 15.7 & 14.3 & 15.1 & 15.5 & 17.2 \\
\hline Materials and supplies & 9.2 & $\ldots$ & $\ldots$ & $\ldots$ & $\cdots$ & $\ldots$ \\
\hline Transfers to the civil service pension fund & 3.2 & $\cdots$ & $\cdots$ & $\cdots$ & $\ldots$ & $\cdots$ \\
\hline Other transfers & 3.5 & $\ldots$ & $\ldots$ & $\ldots$ & $\ldots$ & $\ldots$ \\
\hline Interest payments & 17.1 & 17.1 & 15.9 & 16.2 & 15.7 & 14.8 \\
\hline Domestic & 10.7 & 11.5 & 10.3 & 10.7 & 10.8 & 11.1 \\
\hline Foreign & 6.4 & 5.6 & 5.6 & 5.5 & 5.0 & 3.7 \\
\hline Food subsidies & 5.9 & 7.4 & 7.1 & 8.2 & 4.3 & 3.4 \\
\hline From budgetary contribution & 2.8 & 3.7 & 4.3 & 4.7 & 2.3 & 2.0 \\
\hline From earmarked tariffs & 3.1 & $\ldots$ & $\cdots$ & $\ldots$ & $\cdots$ & $\cdots$ \\
\hline Transfers to local governments & 5.5 & 5.8 & 5.4 & 5.6 & 5.8 & 6.0 \\
\hline Capital expenditure and transfers & 16.8 & 15.9 & 22.4 & 18.4 & 18.3 & 17.8 \\
\hline Central government investment & 13.4 & $\cdots$ & $\cdots$ & $\ldots$ & $\ldots$ & $\ldots$ \\
\hline Capital transfers to public enterprises & 2.2 & $\cdots$ & $\cdots$ & $\cdots$ & $\cdots$ & $\ldots$ \\
\hline Fonds hassan II expenditures & & & & & & \\
\hline Road Fund expenditures & 1.2 & 0.8 & 0.8 & 1.1 & 0.7 & 0.8 \\
\hline
\end{tabular}

Source: Ministry of Finance.

1/ Transition fiscal year July-December 2000. 
Table 16. Morocco: Functional Classification of Central Government Current Expenditure, 1998/99--2002

\begin{tabular}{|c|c|c|c|c|c|}
\hline & & & 2nd half & & Prel. \\
\hline & 1998/99 & $1999 / 00$ & $20001 /$ & 2001 & 2002 \\
\hline & \multicolumn{5}{|c|}{ (In millions of Moroccan dirhams) } \\
\hline Total & 80,664 & 86,459 & 43,869 & 90,412 & 90,691 \\
\hline General public services & 5,007 & 5,427 & 2,841 & 5,714 & 6,012 \\
\hline Defense & 12,416 & 12,284 & 6,589 & 13,443 & 15,191 \\
\hline Public order & 6,916 & 7,556 & 3,739 & 7,840 & 8,879 \\
\hline Education & 18,658 & 19,046 & 10,248 & 21,784 & 23,857 \\
\hline Health & 2,991 & 4,048 & 1,787 & 4,011 & 4,325 \\
\hline Housing & 264 & 382 & 212 & 449 & 483 \\
\hline Recreation, cultural affairs, etc. & 502 & 485 & 267 & 546 & 572 \\
\hline Agriculture & 1,479 & 1,538 & 772 & 1,735 & 1,964 \\
\hline Mines and energy & 151 & 153 & 80 & 177 & 202 \\
\hline Transport and communications & 782 & 819 & 413 & 890 & 1,055 \\
\hline General expenditure 2/ & 9,165 & 10,470 & 6,010 & 16,890 & 11,355 \\
\hline \multirow[t]{2}{*}{ Other } & 22,333 & 24,251 & 14,272 & 16,934 & 16,797 \\
\hline & \multicolumn{5}{|c|}{ (In percent of total expenditure) } \\
\hline Total & 100.0 & 100.0 & 100.0 & 100.0 & 100.0 \\
\hline General public services & 6.2 & 6.3 & 6.5 & 6.3 & 6.6 \\
\hline Defense & 15.4 & 14.2 & 15.0 & 14.9 & 16.7 \\
\hline Public order & 8.6 & 8.7 & 8.5 & 8.7 & 9.8 \\
\hline Education & 23.1 & 22.0 & 23.4 & 24.1 & 26.3 \\
\hline Health & 3.7 & 4.7 & 4.1 & 4.4 & 4.8 \\
\hline Housing & 0.3 & 0.4 & 0.5 & 0.5 & 0.5 \\
\hline Recreation, cultural affairs, etc. & 0.6 & 0.6 & 0.6 & 0.6 & 0.6 \\
\hline Agriculture & 1.8 & 1.8 & 1.8 & 1.9 & 2.2 \\
\hline Mines and energy & 0.2 & 0.2 & 0.2 & 0.2 & 0.2 \\
\hline Transport and communications & 1.0 & 0.9 & 0.9 & 1.0 & 1.2 \\
\hline General expenditure $1 /$ & 11.4 & 12.1 & 13.7 & 18.7 & 12.5 \\
\hline \multirow[t]{2}{*}{ Other } & 27.7 & 28.0 & 24.9 & 18.7 & 18.5 \\
\hline & \multicolumn{5}{|c|}{ (In percent of GDP) } \\
\hline Total & 23.5 & 25.0 & 25.1 & 23.6 & 22.2 \\
\hline General public services & 1.5 & 1.6 & 1.6 & 1.5 & 1.5 \\
\hline Defense & 3.6 & 3.5 & 3.8 & 3.5 & 3.7 \\
\hline Public order & 2.0 & 2.2 & 2.1 & 2.0 & 2.2 \\
\hline Education & 5.4 & 5.5 & 5.9 & 5.7 & 5.8 \\
\hline Health & 0.9 & 1.2 & 1.0 & 1.0 & 1.1 \\
\hline Housing & 0.1 & 0.1 & 0.1 & 0.1 & 0.1 \\
\hline Recreation, cultural affairs, etc. & 0.1 & 0.1 & 0.2 & 0.1 & 0.1 \\
\hline Agriculture & 0.4 & 0.4 & 0.4 & 0.5 & 0.5 \\
\hline Mines and energy & 0.0 & 0.0 & 0.0 & 0.0 & 0.0 \\
\hline Transport and communications & 0.2 & 0.2 & 0.2 & 0.2 & 0.3 \\
\hline General expenditure $1 /$ & 2.7 & 3.0 & 3.4 & 4.4 & 2.8 \\
\hline Other & 6.5 & 7.0 & 6.2 & 4.4 & 4.1 \\
\hline
\end{tabular}

Sources: Ministry of Finance; and Fund staff estimates.

1/ Transition fiscal year July-December 2000.

2/ Outlays for the benefit of all government units (dépenses communes). 
Table 17. Morocco: Functional Classification of Central Government Investment Expenditure, 1998/99-2002

\begin{tabular}{|c|c|c|c|c|c|}
\hline & $1998 / 99$ & $1999 / 00$ & $\begin{array}{l}\text { 2nd half } \\
20001 /\end{array}$ & 2001 & $\begin{array}{l}\text { Prel. } \\
2002\end{array}$ \\
\hline & \multicolumn{5}{|c|}{ (In millions of Moroccan dirhams) } \\
\hline Total & 17,428 & 17,485 & 13,648 & 21,866 & 21,123 \\
\hline General public services & 584 & 820 & 1,708 & 944 & 873 \\
\hline Defense & 1,592 & 1,940 & 1,283 & 2,200 & 1,803 \\
\hline Public order & 886 & 1,130 & 655 & 1,326 & 1,217 \\
\hline Education & 1,330 & 1,934 & 1,331 & 1,992 & 2,037 \\
\hline Health, social security, and welfare & 648 & 925 & 516 & 943 & 858 \\
\hline Housing & 286 & 305 & 385 & 544 & 486 \\
\hline Recreation, cultural affairs, etc. & 103 & 209 & 176 & 316 & 358 \\
\hline Agriculture & 1,878 & 2,234 & 1,160 & 2,284 & 2,748 \\
\hline Mines and energy & 290 & 309 & 167 & 228 & 193 \\
\hline Transport and communications & 3,100 & 3,101 & 1,746 & 2,562 & 2,549 \\
\hline \multirow[t]{2}{*}{ Other } & 6,731 & 4,578 & 3,519 & 8,527 & 8,000 \\
\hline & \multicolumn{5}{|c|}{ (In percent of total) } \\
\hline Total & 100.0 & 100.0 & 100.0 & 100.0 & 100.0 \\
\hline General public services & 3.4 & 4.7 & 12.5 & 4.3 & 4.1 \\
\hline Defense & 9.1 & 11.1 & 9.4 & 10.1 & 8.5 \\
\hline Public order & 5.1 & 6.5 & 4.8 & 6.1 & 5.8 \\
\hline Education & 7.6 & 11.1 & 9.8 & 9.1 & 9.6 \\
\hline Health & 3.7 & 5.3 & 3.8 & 4.3 & 4.1 \\
\hline Housing & 1.6 & 1.7 & 2.8 & 2.5 & 2.3 \\
\hline Recreation, cultural affairs, etc. & 0.6 & 1.2 & 1.3 & 1.4 & 1.7 \\
\hline Agriculture & 10.8 & 12.8 & 8.5 & 10.4 & 13.0 \\
\hline Mines and energy & 1.7 & 1.8 & 1.2 & 1.0 & 0.9 \\
\hline Transport and communications & 17.8 & 17.7 & 12.8 & 11.7 & 12.1 \\
\hline \multirow[t]{2}{*}{ Other } & 38.6 & 26.2 & 33.1 & 39.0 & 37.9 \\
\hline & \multicolumn{5}{|c|}{ (In percent of GDP) } \\
\hline Total & 5.1 & 5.0 & 7.8 & 5.7 & 5.2 \\
\hline Gencral public services & 0.2 & 0.2 & 1.0 & 0.2 & 0.2 \\
\hline Defense & 0.5 & 0.6 & 0.7 & 0.6 & 0.4 \\
\hline Public order & 0.3 & 0.3 & 0.4 & 0.3 & 0.3 \\
\hline Education & 0.4 & 0.6 & 0.8 & 0.5 & 0.5 \\
\hline Health & 0.2 & 0.3 & 0.3 & 0.2 & 0.2 \\
\hline Housing & 0.1 & 0.1 & 0.2 & 0.1 & 0.1 \\
\hline Recreation, cultural affairs, etc. & 0.0 & 0.1 & 0.1 & 0.1 & 0.1 \\
\hline Agriculture & 0.5 & 0.6 & 0.7 & 0.6 & 0.7 \\
\hline Mines and energy & 0.1 & 0.1 & 0.1 & 0.1 & 0.0 \\
\hline Transport and communications & 0.9 & 0.9 & 1.0 & 0.7 & 0.6 \\
\hline Other & 2.0 & 1.3 & 2.6 & 2.2 & 2.0 \\
\hline
\end{tabular}

Sources: Ministry of Finance; and Fund staff estimates.

1/ Transition fiscal year July-December 2000. 
Table 18. Morocco: Functional Classification of Central Government Total Expenditure, 1998/99-2002

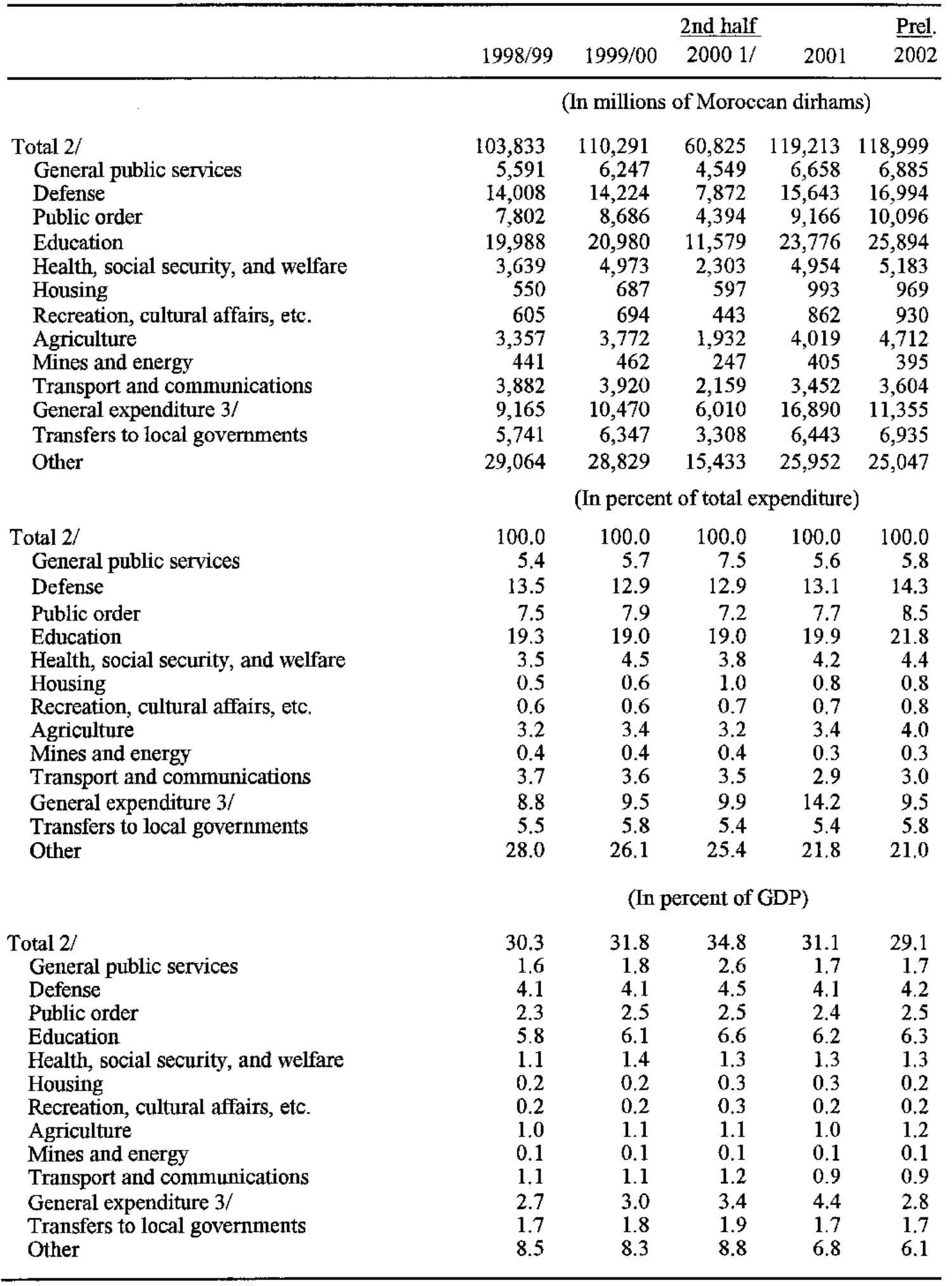

Sources: Ministry of Finance; and Fund staff estimates.

1/ Transition fiscal year July-December 2000.

2/ Excluding net lending.

3/ Outlays for the benefit of all government units (dépenses communes). 
Table 19. Morocco: Administrative Classification of Central Government

Current Expenditure, 1998/99-2002

(In millions of Moroccan dirhams)

\begin{tabular}{lrrrrrr}
\hline & & \multicolumn{5}{c}{ Budget Law } \\
\cline { 3 - 7 } & $1998 / 99$ & $1999 / 00$ & $\frac{2 \text { 2nd half }}{20001 /}$ & 2001 & 2001 & 2002 \\
\hline & & & & & & \\
Total & 80,664 & 84,631 & 47,229 & 45,305 & 93,377 & 96,091 \\
& & & & & & \\
Sovereign & 1,917 & 1,890 & 1,002 & 1,983 & 1,983 & 2,003 \\
Foreign affairs and cooperation & 1,105 & 1,316 & 705 & 908 & 908 & 832 \\
Interior & 5,560 & 5,944 & 2,968 & 6,264 & 6,264 & 7,151 \\
Justice & 1,356 & 1,612 & 771 & 1,576 & 1,576 & 1,728 \\
Public works & 626 & 639 & 322 & 708 & 708 & 854 \\
Finance & 1,211 & 1,331 & 653 & 1,378 & 1,378 & 1,583 \\
Agriculture & 1,479 & 1,538 & 772 & 1,735 & 1,735 & 1,964 \\
Education & 18,658 & 19,046 & 10,248 & 21,784 & 21,784 & 23,857 \\
Health & 1,991 & 4,048 & 1,787 & 4,011 & 4,011 & 4,325 \\
Defense & 14,416 & 12,284 & 6,589 & 13,443 & 13,443 & 15,191 \\
General expenditure $2 /$ & 9,165 & 10,470 & 6,010 & 16,890 & 16,890 & 11,355 \\
Other 3/ & 23,180 & 24,513 & 15,402 & $-25,375$ & 22,697 & 25,248 \\
\hline & & & & & & \\
\hline
\end{tabular}

Source: Ministry of Finance.

1/ Transition fiscal year July-December 2000.

2/ Outlays, mainly for utilities, for the benefit of all government units (dépenses communes).

$3 /$ Includes interest payments. For the 1999/00 budget, also includes contingent allocation for unexpected expenditure. 
Table 20. Morocco: Administrative Classification of Central Government Investment Expenditure, 1998/99-2002

(In millions of Moroccan dirhams)

\begin{tabular}{lrrrrr}
\hline & & \multicolumn{5}{c}{ Budget Law } \\
\cline { 3 - 6 } & $1998 / 99$ & $1999 / 00$ & $\frac{2 \text { nd half }}{20001 /}$ & 2001 & 2002 \\
\hline & & & & & \\
Total & 17,428 & 17,950 & 12,146 & 19,790 & 18,655 \\
Sovereign & & & & & \\
Prime Minister & 222 & 222 & 111 & 222 & 174 \\
Defense & 0 & 0 & 1,200 & 0 & 0 \\
Interior & 1,592 & 1,940 & 1,283 & 2,200 & 1,803 \\
Finance & 756 & 869 & 500 & 1,020 & 946 \\
Energy and mining & 4,723 & 4,641 & 3,206 & 7,277 & 5,432 \\
Agriculture & 290 & 309 & 167 & 228 & 193 \\
Public works & 1,878 & 2,234 & 1,160 & 2,285 & 2,748 \\
Transport & 2,381 & 2,380 & 1,378 & 2,515 & 2,502 \\
Education & 716 & 716 & 360 & 41 & 36 \\
Health & 1,330 & 1,934 & 1,331 & 1,992 & 2,037 \\
Housing & 648 & 925 & 516 & 943 & 858 \\
Other & 286 & 305 & 385 & 544 & 486 \\
& 2,606 & 1,475 & 549 & 523 & 1,440 \\
\hline
\end{tabular}

Source: Ministry of Finance.

1/ Transition fiscal year July-December 2000.

2/ Outlays, mainly for utilities, for the benefit of all government units (dépenses communes). 
Table 21. Morocco: Central Government Employment and Wage Indicators, 1998/99-2002

\begin{tabular}{lrrrrr}
\hline & & & 2nd half & Prel. \\
& $1998 / 99$ & $1999 / 00$ & $20001 /$ & 2001 & 2002 \\
\hline Employment (number) & & & & & \\
$\quad$ Annual change in percent & 770,723 & 782,494 & 796,038 & 808,225 & 819,282 \\
& 1.0 & 1.5 & 1.7 & 1.5 & 1.4 \\
Wage bill (in millions of dirhams) & 40,286 & 42,132 & 21,222 & 47,998 & 48,580 \\
$\quad$ Annual change in percent & 7.2 & 4.6 & 0.7 & 13.1 & 1.2 \\
& & & & & \\
Average wage (in dirhams per annum) & 52,270 & 53,843 & 53,319 & 59,387 & 59,296 \\
Average real wage (1989 dirhams) & 33,974 & 34,544 & 33,885 & 37,511 & 36,434 \\
Annual change (in percent) & & & & & \\
$\quad$ Nominal wage & 6.1 & 3.0 & -1.0 & 11.4 & -0.2 \\
$\quad$ Real wage & 4.3 & 1.7 & -1.9 & 10.7 & -2.9 \\
$\quad$ Ratio of wage bill to GDP & 11.8 & 12.2 & 12.1 & 12.5 & 11.9 \\
$\quad$ & & & & & \\
Memorandum items: & 27.8 & 28.2 & 28.7 & 29.2 & 29.5 \\
$\quad$ Population (in millions) 2/ & 153.9 & 155.9 & 157.4 & 158.3 & 162.7 \\
$\quad$ CPI index (1989 = 100) 3/ & 1.7 & 1.3 & 1.0 & 0.6 & 2.8 \\
$\quad$ Average CPI increase (in percent) & & & & & \\
\hline
\end{tabular}

Sources: Ministry of Finance; and Fund staff estimates.

1/ Transition fiscal year July-December 2000.

2/ Calendar year.

3/ Average for fiscal years through 1999/2000; calendar year from 2001. 
Table 22. Morocco: Central Government Debt, 1998/99-2002 1/

(In millions of Moroccan dirhams)

\begin{tabular}{lrrrrr}
\hline & & & & & Prel. \\
& $1998 / 99$ & $1999 / 00$ & $20002 /$ & 2001 & 2002 \\
\hline & & & & & \\
Stock at end-period & 264,997 & 259,674 & 268,034 & 286,483 & 284,145 \\
External debt & 128,276 & 120,186 & 118,646 & 110,597 & 92,800 \\
Domestic debt 3/ & 136,721 & 139,488 & 149,388 & 175,886 & 196,554 \\
$\quad$ Long-term & 73,589 & 68,798 & 70,680 & 70,863 & 81,652 \\
Medium-term & 45,993 & 46,571 & 51,618 & 53,605 & 62,032 \\
Short-term & 17,140 & 24,119 & 27,090 & 51,418 & 52,870 \\
& & & & & \\
Drawings & 53,875 & 49,930 & 32,402 & 77,876 & 98,983 \\
External debt & 8,665 & 4,816 & 3,810 & 3,150 & 3,984 \\
Domestic debt 3/ & 45,210 & 45,114 & 28,592 & 74,726 & 94,999 \\
Long-term & 13,783 & 3,481 & 3,516 & 3,261 & 14,306 \\
Medium-term & 11,904 & 7,104 & 7,017 & 12,928 & 22,582 \\
Short-term & 19,523 & 34,169 & 18,059 & 58,536 & 63,087 \\
& & & & & \\
Amortization & 46,843 & 54,840 & 24,098 & 61,432 & 95,002 \\
External debt & 13,856 & 12,352 & 5,546 & 13,203 & 15,683 \\
Domestic debt 3/ & 32,987 & 42,488 & 18,552 & 48,229 & 79,319 \\
$\quad$ Long-term & 5,355 & 8,631 & 1,635 & 3,078 & 3,529 \\
Medium-term & 4,263 & 6,526 & 1,970 & 10,941 & 14,155 \\
Short-term & 23,369 & 27,331 & 14,947 & 34,210 & 61,635 \\
\hline
\end{tabular}

Source: Ministry of Finance and Fund staff estimates

1/ Remunerated debt only.

2/ Transition fiscal year July-December 2000.

3/ By initial maturities. 
Table 23. Morocco: External Debt Service of Central Government, 1998/99-2002

(In millions of Moroccan dirhams)

\begin{tabular}{lrrrrr}
\hline & & & $\frac{2 \text { 2nd half }}{20001 /}$ & 2001 & $\frac{\text { Prel. }}{2002}$ \\
\hline Debt service & $1998 / 99$ & $1999 / 00$ & & & \\
$\quad$ Interest & 20,499 & 18,547 & 9,439 & 19,111 & 20,120 \\
Amortization & 6,643 & 6,195 & 3,393 & 5,908 & 4,437 \\
& 13,856 & 12,352 & 6,046 & 13,203 & 15,683 \\
\hline
\end{tabular}

Source: Ministry of Finance.

1/ Transition fiscal year July-December 2000. 
Table 24. Morocco: Current Subsidies to Public Enterprises, 1998/99-2002

(In millions of Moroccan dirhams)

\begin{tabular}{lrrrrr}
\hline & & & $\underline{2 \text { nd half }}$ & $\underline{\underline{\text { Prel }}}$ \\
& $1998 / 99$ & $1999 / 00$ & $20001 /$ & 2001 & 2002 \\
\hline Total & 1,553 & 1,657 & 816 & 1817 & 1922 \\
& & & & & \\
ORMVA (agricultural development) 2/ & 139 & 151 & 68 & 207 & 254 \\
Centres de travaux (public works) & 208 & 209 & 103 & 225 & 271 \\
Other 3/ & 1,206 & 1,297 & 645 & 1,385 & 1,397 \\
& & & & & \\
\hline
\end{tabular}

Source: Ministry of Finance.

1/ Transition fiscal year July-December 2000.

2/ Offices régionaux de mise en valeur agricole.

3/ Excluding transfers to the Caisse de compensation, ONICL, and the Caisse marocaine des retraites. 
Table 25. Morocco: Capital Subsidies to Public Enterprises, 1998/99-2002

(In millions of Moroccan dirhams)

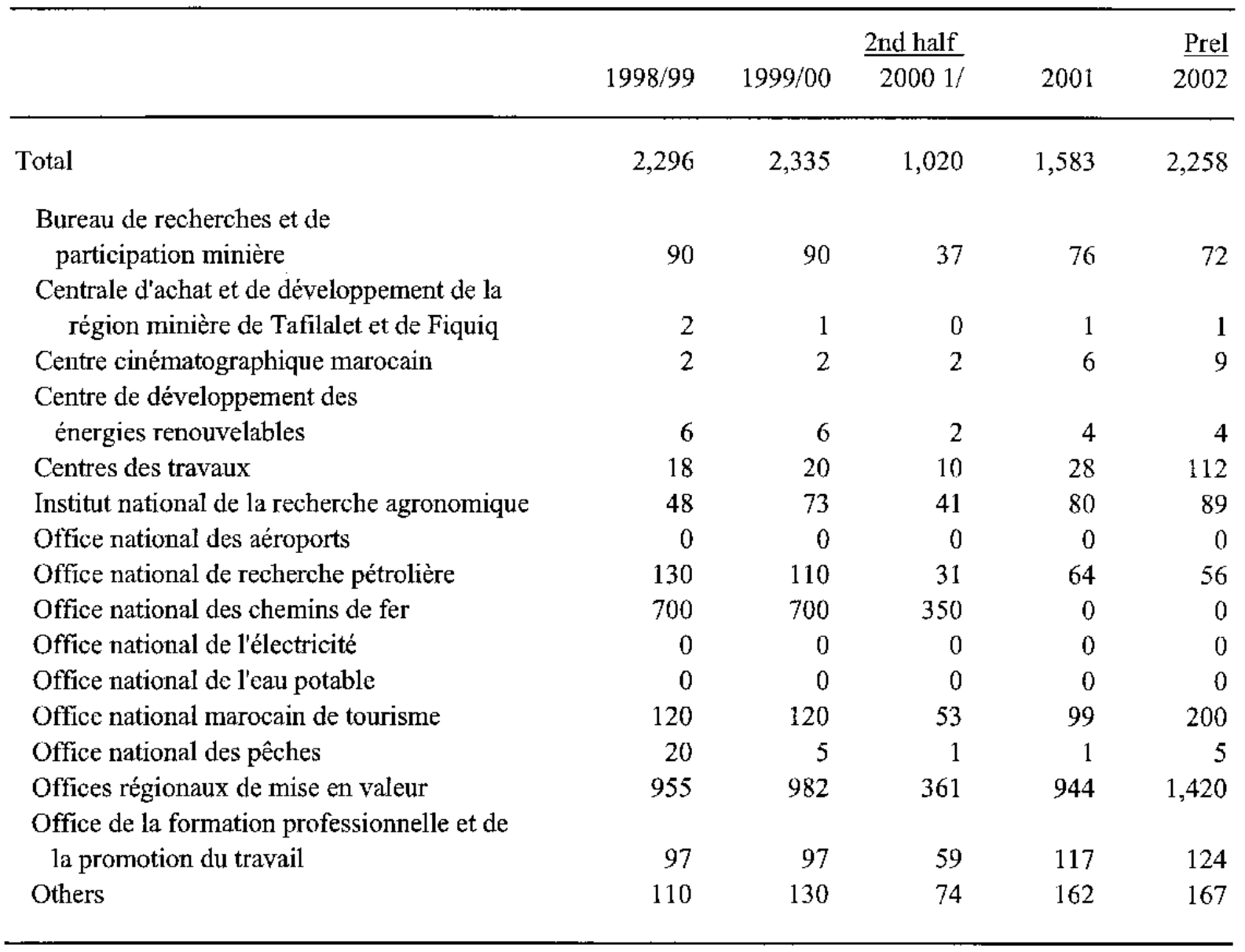

Source: Ministry of Finance.

1/ Transition fiscal year July-December 2000. 
Table 26. Morocco: Monetary Survey, 1998-2002

\begin{tabular}{|c|c|c|c|c|c|}
\hline & 1998 & 1999 & 2000 & 2001 & 2002 \\
\hline & \multicolumn{5}{|c|}{ (In billions of dirahms) } \\
\hline Net foreign assets & 43.4 & 59.6 & 55.3 & 102.6 & 111.3 \\
\hline Net domestic assets & 196.2 & 204.7 & 231.3 & 224.8 & 236.9 \\
\hline Domestic credit & 260.0 & 268.2 & 293.0 & 294.3 & 306.7 \\
\hline Net credit to the government & 91.5 & 82.2 & 92.4 & 85.6 & 89.5 \\
\hline Credit to the economy & 168.5 & 185.9 & 200.6 & 208.6 & 217.3 \\
\hline Money and quasi money & 239.6 & 264.3 & 286.6 & 327.4 & 348.2 \\
\hline Money & 174.5 & 194.9 & 210.3 & 243.1 & 264.9 \\
\hline Currency outside banks & 50.6 & 56.7 & 58.2 & 66.0 & 69.6 \\
\hline Demand deposits & 123.8 & 138.2 & 152.1 & 177.1 & 195.3 \\
\hline Quasi money & 65.1 & 69.4 & 76.3 & 84.3 & 83.3 \\
\hline \multirow[t]{2}{*}{ Other liabilities, net } & 63.8 & 63.5 & 61.7 & 69.5 & 69.9 \\
\hline & \multicolumn{5}{|c|}{ (12-month percent changc) } \\
\hline Net domestic assets & 5.9 & 4.3 & 13.0 & -2.8 & 5.4 \\
\hline Domestic credit & 6.0 & 3.1 & 9.3 & 0.4 & 4.2 \\
\hline Net credit to the government & -2.0 & -10.1 & 12.4 & -7.4 & 4.5 \\
\hline Credit to the economy & 10.8 & 10.3 & 7.9 & 4.0 & 4.1 \\
\hline \multirow[t]{2}{*}{ Money and quasi money } & 5.8 & 10.3 & 8.4 & 14.2 & 6.3 \\
\hline & \multicolumn{5}{|c|}{ (In percent of broad money; in beginning of period) } \\
\hline Net foreign assets & 1.0 & 6.8 & -1.6 & 16.5 & 2.6 \\
\hline Domestic credit & 6.5 & 3.4 & 9.4 & 0.4 & 3.8 \\
\hline Net credit to the government & -0.8 & -3.9 & 3.9 & -2.4 & 1.2 \\
\hline Credit to the economy & 7.3 & 7.3 & 5.5 & 2.8 & 2.6 \\
\hline Other assets net & -1.6 & 0.1 & 0.7 & -2.7 & -0.1 \\
\hline \multicolumn{6}{|l|}{ Memorandum items: } \\
\hline Velocity (GDP/M3) & 1.44 & 1.31 & 1.24 & 1.17 & 1.18 \\
\hline Velocity (non-agr. GDP/M3) & 1.18 & 1.11 & 1.06 & 0.98 & 0.98 \\
\hline Money multiplier & 3.59 & 3.42 & 3.60 & 3.35 & 3.39 \\
\hline Credit to economy/GDP & 49.0 & 53.8 & 56.6 & 54.5 & 53.1 \\
\hline Credit to economy/non-agro GDP & 59.2 & 63.5 & 65.9 & 64.7 & 63.4 \\
\hline
\end{tabular}

Sources: Bank Al-Maghrib; and Fund staff estimates. 
Table 27. Morocco: Summary Accounts of Bank Al-Maghrib, 1998-2002

\begin{tabular}{|c|c|c|c|c|c|}
\hline & 1998 & 1999 & 2000 & 2001 & 2002 \\
\hline & \multicolumn{5}{|c|}{ (In millions of dirhams) } \\
\hline Foreign assets & 42,674 & 58,416 & 52,250 & 98,897 & 104,005 \\
\hline \multicolumn{6}{|l|}{ Of which: } \\
\hline Gold holdings & 1,950 & 1,950 & 1,955 & 1,957 & 1,960 \\
\hline Gross reserves & 40,437 & 56,181 & 50,010 & 96,640 & 101,760 \\
\hline Net claims on the government & 26,634 & 20,597 & 23,504 & 5,187 & 5,163 \\
\hline Claims on the deposit money banks & 3,381 & 1,346 & 7,161 & 7 & 1 \\
\hline Assets $=$ liabilities & 72,689 & 80,359 & 82,915 & 104,091 & 109,169 \\
\hline Reserve money & 66,965 & 77,260 & 79,616 & 97,664 & 102,627 \\
\hline Currency outside banks & 50,644 & 56,713 & 58,169 & 66,025 & 69,565 \\
\hline Bank reserves & 13,937 & 17,558 & 19,658 & 29,070 & 29,817 \\
\hline Other deposits & 2,384 & 2,989 & 1,789 & 2,569 & 3,245 \\
\hline Foreign liabilities & 725 & 826 & 893 & 927 & 809 \\
\hline SDR allocation & 434 & 1,157 & 1,169 & 1,171 & 1,173 \\
\hline Other items, net & 5,290 & 1,942 & 2,130 & 5,246 & 5,352 \\
\hline \multicolumn{6}{|l|}{ Memorandum item: } \\
\hline \multirow[t]{2}{*}{ Treasury cash holdings } & 825 & 532 & 806 & 10,806 & 11,322 \\
\hline & & \multicolumn{4}{|c|}{ (12-month percent change) } \\
\hline Net claims on the government & -1.6 & -22.7 & 14.1 & -77.9 & -0.5 \\
\hline Claims on the deposit money banks & 179.6 & -60.2 & 432.0 & -99.9 & -85.7 \\
\hline \multirow[t]{2}{*}{ Reserve money } & 6.1 & 15.4 & 3.0 & 22.7 & 5.1 \\
\hline & & \multicolumn{4}{|c|}{ (In percent of reserve money) } \\
\hline Foreign assets & 63.7 & 75.6 & 65.6 & 101.3 & 101.3 \\
\hline Net claims on the government & 39.8 & 26.7 & 29.5 & 5.3 & 5.0 \\
\hline Claims on the deposit money banks & 5.0 & 1.7 & 9.0 & 0.0 & 0.0 \\
\hline Reserve money & 100.0 & 100.0 & 100.0 & 100.0 & 100.0 \\
\hline Currency outside banks & 75.6 & 73.4 & 73.1 & 67.6 & 67.8 \\
\hline Bank reserves & 20.8 & 22.7 & 24.7 & 29.8 & 29.1 \\
\hline Other deposits & 3.6 & 3.9 & 2.2 & 2.6 & 3.2 \\
\hline
\end{tabular}

Source: Bank Al-Maghrib.

1/ Starting in 1998, the category deposit money banks includes BNDE, CNCA, and CIH. 
Table 28. Morocco: Summary Accounts of the Deposit Money Banks, 1998-2002 1/

\begin{tabular}{|c|c|c|c|c|c|}
\hline & 1998 & 1999 & 2000 & 2001 & 2002 \\
\hline & & \multicolumn{4}{|c|}{ (In millions of dirahms) } \\
\hline Reserves $2 /$ & 13,937 & 17,558 & 19,658 & 29,070 & 29,817 \\
\hline Foreign assets $3 /$ & 4,592 & 4,812 & 6,356 & 6,566 & 9,040 \\
\hline Net claims on the government & 58,615 & 54,917 & 61,729 & 73,161 & 76,933 \\
\hline Credit to the private sector & 168,495 & 185,905 & 200,551 & 208,647 & 217,269 \\
\hline Assets $=$ liabilities & 245,639 & 263,192 & 288,294 & 317,444 & 333,059 \\
\hline Deposits & 179,899 & 196,852 & 218,446 & 250,532 & 266,987 \\
\hline Demand & 114,785 & 127,463 & 142,165 & 166,238 & 183,682 \\
\hline Time & 65,114 & 69,389 & 76,281 & 84,294 & 83,305 \\
\hline Foreign liabilities & 4,276 & 4,605 & 4,322 & 3,881 & 2,740 \\
\hline Credit from the central bank & 3,381 & 1,346 & 7,161 & 7 & 1 \\
\hline Capital & 26,937 & 29,106 & 29,480 & 32,574 & 32,477 \\
\hline Provisions & 12,944 & 14,916 & 17,271 & 22,037 & 25,150 \\
\hline Other items (net) & 18,202 & 16,367 & 11,614 & 8,413 & 5,704 \\
\hline \multicolumn{6}{|l|}{ Related data } \\
\hline \multirow[t]{2}{*}{ Private sector claims on government } & 6,652 & 7,711 & 8,172 & 8,285 & 8,358 \\
\hline & & \multicolumn{4}{|c|}{ (Percentage change) } \\
\hline Reserves & 13.3 & 26.0 & 12.0 & 47.9 & 2.6 \\
\hline Foreign assets & 24.0 & 4.8 & 32.1 & 3.3 & 37.7 \\
\hline Net claims on the government & 0.0 & -6.3 & 12.4 & 18.5 & 5.2 \\
\hline Credit to the private sector & 10.8 & 10.3 & 7.9 & 4.0 & 4.1 \\
\hline Deposits & 7.4 & 9.4 & 11.0 & 14.7 & 6.6 \\
\hline Demand & 11.0 & 11.0 & 11.5 & 16.9 & 10.5 \\
\hline Time & 1.5 & 6.6 & 9.9 & 10.5 & -1.2 \\
\hline Foreign liabilities & 25.4 & 7.7 & -6.1 & -10.2 & -29.4 \\
\hline Problem loans (in millions of dirhams) & 21,144 & 28,560 & 30,927 & 35,699 & 38,765 \\
\hline Problem loans in percent of total credit to economy & 13 & 15 & 15 & 17 & 18 \\
\hline Total provisions as percent of problem loans & 61 & 52 & 56 & 62 & 65 \\
\hline Capital as percent of total assets & 11 & 11 & 10 & 10 & 10 \\
\hline
\end{tabular}

Source: Bank Al-Maghrib.

1/ Starting in 1998, the category deposit money banks includes BNDE, CNCA, and $\mathrm{CIH}$.

2/ Including forex deposits with Bank Al-Maghrib.

3/ Excluding forex deposits with Bank Al-Maghrib. 
Table 29. Morocco: Merchandise Exports, f.o.b., 1998-2001 1/

(In millions of U.S. dollars)

\begin{tabular}{|c|c|c|c|c|}
\hline & 1998 & 1999 & 2000 & 2001 \\
\hline Food, beverages, and tobacco & 1,484 & 1,515 & 1,576 & 1,482 \\
\hline Citrus fruits & 270 & 267 & 185 & 184 \\
\hline Vegetables & 273 & 323 & 234 & 253 \\
\hline Tomatoes & 121 & 124 & 76 & 82 \\
\hline Potatoes & 13 & 32 & 17 & 13 \\
\hline Other fresh vegetables & 42 & 54 & 54 & 66 \\
\hline Canned vegetables & 97 & 114 & 86 & 91 \\
\hline Other & 942 & 926 & 1,157 & 1,045 \\
\hline Energy and lubricants & 104 & 203 & 271 & 301 \\
\hline Agricultural raw materials & 143 & 188 & 170 & 123 \\
\hline Raw minerals & 639 & 610 & 543 & 507 \\
\hline Phosphates & 459 & 455 & 389 & 373 \\
\hline Lead & 18 & 18 & 10 & 9 \\
\hline Barium sulphate & 16 & 9 & 12 & 17 \\
\hline Other & 145 & 128 & 132 & 109 \\
\hline Semifinished products & 1,301 & 1,396 & 1,603 & 1,531 \\
\hline Fertilizers & 343 & 317 & 323 & 349 \\
\hline Phosphoric acid & 498 & 589 & 505 & 449 \\
\hline Lead & 32 & 28 & 20 & 27 \\
\hline Other & 428 & 462 & 755 & 705 \\
\hline Manufactured goods & 3,472 & 3,598 & 3,256 & 3,197 \\
\hline Industrial equipment & 548 & 693 & 450 & 399 \\
\hline Consumer goods & 2,924 & 2,905 & 2,806 & 2,798 \\
\hline Total exports (customs basis) & 7,143 & 7,508 & 7,419 & 7,142 \\
\hline \multicolumn{5}{|l|}{ Memorandum items: } \\
\hline Phosphates and derivatives & 1,300 & 1,361 & 1,216 & 1,171 \\
\hline All other & 5,843 & 6,147 & 6,202 & 5,971 \\
\hline Recxports, under admission temporaire $2 /$ & 2,477 & 2,644 & 2,558 & 2,438 \\
\hline
\end{tabular}

Source: Office des Changes.

1/ Excluding trade under the admission temporaire sans paiement regime until 1997, for which a commodity breakdown is not available.

2/ Included in the commodity breakdown since 1998. 
Table 30. Morocco: Banks' Interest Rates, 1998-2002

(In percent per annum; end of period)

\begin{tabular}{lrrrrr}
\hline & 1998 & 1999 & 2000 & 2001 & 2002 \\
\hline & & & & & \\
Deposits rates & & & & & \\
$\quad$ 6-month deposits 1/ & 6.3 & 5.1 & 5.0 & 4.5 & 3.6 \\
$\quad$ 12-month deposits 1/ & 6.8 & 5.7 & 5.1 & 4.8 & 4.0 \\
& & & & & \\
Lending rates & & & & & \\
$\quad$ Short term & 7.8 & 7.0 & 7.0 & 7.0 & 7.0 \\
$\quad$ Observed minimum & 14.0 & 13.5 & 13.3 & 13.3 & 13.3 \\
$\quad$ Observed maximum & 8.0 & 8.0 & 7.0 & 8.0 & 8.0 \\
$\quad$ Long term & 14.0 & 13.5 & 13.3 & 13.3 & 13.3 \\
$\quad$ Observed minimum & & & & & \\
$\quad$ Observed maximum & & & & \\
\hline
\end{tabular}

Source: Bank Al-Maghrib.

$1 /$ Monthly averages. 
Table 31. Morocco: Balance of Payments, 1998-2002

(In millions of U.S. dollars; unless otherwise indicated)

\begin{tabular}{|c|c|c|c|c|c|}
\hline & 1998 & 1999 & 2000 & 2001 & $\frac{\text { Prel. }}{2002}$ \\
\hline Trade balance & $-2,323$ & $-2,448$ & $-3,235$ & $-3,022$ & $-2,956$ \\
\hline Exports, f.o.b. & 7,143 & 7,508 & 7,419 & 7,142 & 7,708 \\
\hline Phosphates and derived products & 1,300 & 1,361 & 1,216 & 1,171 & 1,198 \\
\hline Imports, f.o.b. & $-9,467$ & $-9,957$ & $-10,653$ & $-10,164$ & $-10,664$ \\
\hline Energy & 922 & 1,326 & 2,038 & 1,945 & 1,808 \\
\hline Services balance & -160 & 124 & 274 & 1,079 & 422 \\
\hline Nonfactor services & 864 & 1,112 & 1,142 & 1,910 & 1,122 \\
\hline Tourism receipts & 1,744 & 1,949 & 2,039 & 2,583 & 2,152 \\
\hline Net investment income & $-1,023$ & -988 & -867 & -831 & -700 \\
\hline Private transfers (net) & 2,293 & 2,144 & 2,382 & 3,535 & 3,532 \\
\hline Official grants (net) & 52 & 10 & 101 & 20 & 83 \\
\hline Current account & -138 & -170 & -478 & 1,612 & 1,082 \\
\hline Capital account $1 /$ & 52 & 259 & 1 & 91 & 86 \\
\hline Financial account & 334 & 1,549 & 63 & 2,144 & -567 \\
\hline Direct investment & 313 & 831 & 368 & 2,727 & 275 \\
\hline Other private (including errors and omissions) & 474 & 1,233 & 536 & 533 & 490 \\
\hline Public medium-and long-term loans (net) & -453 & -515 & -841 & $-1,117$ & $-1,333$ \\
\hline Disbursements & 1,498 & 1,501 & 835 & 628 & 635 \\
\hline Amortization & $-1,951$ & $-2,016$ & $-1,676$ & $-1,745$ & $-1,968$ \\
\hline Reserve assct accumulation (-incrcasc) & -247 & $-1,639$ & 415 & $-3,848$ & -600 \\
\hline \multicolumn{6}{|l|}{ Memorandum items: } \\
\hline Trade balance (in percent of GDP) & -6.5 & -6.9 & -9.7 & -8.9 & -8.0 \\
\hline Current account balance (in percent of GDP) & -0.4 & -0.5 & -1.4 & 4.8 & 2.9 \\
\hline Excluding official grants (in percent of GDP) & -0.5 & -0.5 & -1.7 & 4.7 & 2.7 \\
\hline Gross official reserves $2 /$ & 4,600 & 5,701 & 4,796 & 8,431 & 10,108 \\
\hline $\begin{array}{l}\text { (In months of imports of goods } \\
\text { and nonfactor services) }\end{array}$ & 4.8 & 5.7 & 4.6 & 8.2 & 9.4 \\
\hline $\begin{array}{l}\text { Debt service as percentage of export of goods, } \\
\text { non-factor services and MRE } 3 / 4 /\end{array}$ & 23.9 & 20.8 & 20.1 & 16.2 & 17.3 \\
\hline $\begin{array}{l}\text { External public and publically guarantced } \\
\text { debt (in percent of GDP) }\end{array}$ & 54.0 & 49.8 & 48.2 & 41.5 & 37.5 \\
\hline
\end{tabular}

Sources: Ministry of Finance; Office des Changes; and Fund staff estimates and projections.

1/ Includes the grant element of debt swap operations with France and Spain.

2/ Excluding the reserve position in the Fund.

3/ Public and publically guaranteed debt.

4/ Excluding early amortization on account of debt swaps. 
Table 32. Morocco: Effective Exchange Rates, 1989-2002

(Indices: $1990=100$ )

\begin{tabular}{|c|c|c|c|c|}
\hline & $\begin{array}{l}\text { Nominal Effective } \\
\text { Exchange Ratc } 1 / \\
\text { (1) }\end{array}$ & $\begin{array}{l}\text { Relative Consumer } \\
\text { Prices 2/ } \\
\text { (2) }\end{array}$ & $\begin{array}{l}\text { Real Effective Exchange } \\
\text { Ratc } 1 / \\
(3)=(1) \times(2)\end{array}$ & $\begin{array}{c}\text { Consumer Price } \\
\text { Index }\end{array}$ \\
\hline 1989 & 100.3 & 105.8 & 106.1 & 93.6 \\
\hline 1990 & 100.0 & 100.0 & 100.0 & 99.9 \\
\hline 1991 & 100.9 & 101.4 & 102.4 & 108.9 \\
\hline 1992 & 103.5 & 99.5 & 103.0 & 115.2 \\
\hline 1993 & 109.4 & 96.8 & 105.9 & 121.2 \\
\hline 1994 & 116.6 & 93.7 & 109.3 & 127.5 \\
\hline 1995 & 118.3 & 95.4 & 112.8 & 135.3 \\
\hline 1996 & 119.2 & 95.5 & 113.7 & 139.3 \\
\hline 1997 & 121.4 & 94.5 & 114.7 & 140.7 \\
\hline 1998 & 122.5 & 95.5 & 117.0 & 143.5 \\
\hline 1999 & 124.8 & 95.1 & 118.7 & 145.6 \\
\hline 2000 & 128.9 & 94.7 & 122.0 & 148.4 \\
\hline 2001 & 125.9 & 93.0 & 117.0 & 149.3 \\
\hline 2002 & 124.6 & 93.7 & 116.7 & 153.5 \\
\hline \multicolumn{5}{|l|}{2002} \\
\hline I & 124.6 & 94.5 & 117.6 & 153.4 \\
\hline II & 124.3 & 94.5 & 117.4 & 154.7 \\
\hline III & 124.5 & 93.0 & 115.7 & 152.0 \\
\hline IV & 124.9 & 92.8 & 115.9 & 153.8 \\
\hline \multicolumn{5}{|l|}{2002} \\
\hline January & 124.2 & 93.8 & 116.5 & 152.1 \\
\hline Fcbruary & 124.8 & 94.6 & 118.0 & 153.2 \\
\hline March & 124.7 & 95.0 & 118.4 & 154.9 \\
\hline April & 124.6 & 93.9 & 117.0 & 154.3 \\
\hline May & 124.0 & 95.3 & 118.2 & 156.0 \\
\hline June & 124.3 & 94.1 & 117.0 & 153.9 \\
\hline July & 124.1 & 93.3 & 115.7 & 151.8 \\
\hline August & 124.2 & 92.9 & 115.4 & 151.8 \\
\hline September & 125.1 & 92.8 & 116.1 & 152.3 \\
\hline October & 125.4 & 92.6 & 116.1 & 153.0 \\
\hline November & 124.7 & 93.0 & 115.9 & 154.3 \\
\hline Decemeber & 124.7 & 92.7 & 115.6 & 154.0 \\
\hline
\end{tabular}

Source: IMF, Information Notice System.

1/ Increases equal appreciation.

2/ Domestic price index/weighted partner price index. 
Table 33. Morocco: Stock Exchange Selected Indicators, 1998-2002

\begin{tabular}{|c|c|c|c|c|c|}
\hline & 1998 & 1999 & 2000 & 2001 & $20021 /$ \\
\hline General index & 804 & 777 & 658 & 610 & $\ldots$ \\
\hline Change (in percent) & 20.4 & -3.4 & -15.3 & -7.4 & -16.5 \\
\hline \multicolumn{6}{|l|}{ Market capitalization 2/ } \\
\hline In billions of dirhams & 145.2 & 138.0 & 114.8 & 104.8 & 87.2 \\
\hline In percent of GDP & 42.4 & 40.2 & 32.8 & 27.5 & 21.3 \\
\hline \multicolumn{6}{|l|}{ Trading volume } \\
\hline In billions of $\mathrm{DH}$ & 58.3 & 96.9 & 38.5 & 26.8 & 22.5 \\
\hline Change (in percent) & 80.3 & 66.2 & -60.3 & -30.5 & -66.7 \\
\hline \multicolumn{6}{|c|}{ Share in total transactions of 10 most traded stocks } \\
\hline (in percent) & 77 & 86 & 81 & 79 & 77 \\
\hline Number of listed enterprises & 53 & 54 & 53 & 55 & 55 \\
\hline \multicolumn{6}{|l|}{ Share of transactions off-floor } \\
\hline In percent of total & 71 & 62 & 56 & 35 & 60 \\
\hline Dividend yield (in percent) & $\cdots$ & 2.1 & 3.2 & $\cdots$ & \\
\hline Price earning ratio $(\mathrm{P} / \mathrm{E})$ & $\cdots$ & 17.4 & 12.8 & 8.9 & 8.5 \\
\hline \multicolumn{6}{|l|}{ Memorandum item: } \\
\hline GDP (in billions of $\mathrm{DH}$ ) & 343 & 343 & 350 & 382 & 410 \\
\hline
\end{tabular}

Sources: Ministry of Finance; andConseil Déontologique des Valeurs Mobilières.

1/ New index (Morocco All Shares Index-MASI) starting January 2002.

2/ Excludes the part of equity for which shares are not listed. 
SUMMARY OF THE MOROCCAN TAX SYSTEM (current as of the 2003 Budget Law)

\begin{tabular}{|c|c|c|}
\hline TAX & DESCRIPTION & DEDUCIIONS AND EXEMPTIONS \\
\hline $\begin{array}{l}1 \text { - DIRECT } \\
\text { TAXES } \\
1 / \text { IGR (General } \\
\text { Income Tax) }\end{array}$ & $\begin{array}{l}\text { (1) APPLICABILITY: } \\
\text { The IGR is levied on the income of individuals and } \\
\text { partnerships: } \\
\text { Subject to the tax: } \\
\text { - earned income; } \\
\text { - occupational income; } \\
\text { - income and profits from real estate (1); } \\
\text { - income and profits from invested capital (2); } \\
\text { - agricultural income (3); } \\
\text { A - Earned income: } \\
\quad \quad \text { Definitions: } \\
\text { The following are considered earned income: } \\
\text { salaries; } \\
\text { allowances and fees; } \\
\text { wages; } \\
\text { pensions; } \\
\text { life annuities. } \\
\text { Benefits in cash or in kind paid in addition to the above } \\
\text { income are also considered earned income. }\end{array}$ & $\begin{array}{l}\text { Earned income } \\
\text { Main exemptions } \\
\text { - Family allowances and family assistance payments; } \\
\text { - Alimony; } \\
\text { - Employer's share of retirement and social security contributions; } \\
\text { - Daily illness, accident, and maternity allowances and death benefits; } \\
\text { - Severance pay up to the limit established by the law and regulations in force; } \\
\text { - Disability pensions paid to military personnel and their heirs. } \\
\text { Main deductions } \\
\text { Net taxable wages are obtained by deducting the following major items: } \\
\text { a - 17 percent: for all occupational categories excluding certain occupations (journalists, } \\
\text { miners, merchant marines and seagoing fishermen, etc.) for which the law provides specific } \\
\text { rates. This deduction must not, however, exceed DH } 24,000 ; \\
\text { b- withholding for pensions or retirement funds; } \\
\text { c- contributions to social welfare agencies as well as social security contributions withheld } \\
\text { from wages; } \\
\text { d- the employce's share of group life insurance premiums; } \\
\text { e- repayment of regular principal and interest on loans obtained for the purchase of low- } \\
\text { cost housing } \\
\text { Standard deduction for pensions and life annuities } \\
\text { A deduction of } 40 \text { percent is allowed for pensions and life annuities. } \\
\text { Deduction of the tax on foreign retirement pensions } \\
\text { to a reduction of } 80 \text { percent of the tax payable on the portion of their pensions permanently } \\
\text { transferred to Morocco, in nonconvertiblc dirhams. }\end{array}$ \\
\hline
\end{tabular}

(1) and (2): Effective 01/01/2001: - real estate profits subject to TPI (real estate profits tax), are now subject to IGR, as the TPI was abolished by the 2001 Budget Law (BL) - income and profits from invested capital subject to the TPPRF (tax on fixed-income investment profits), the TPA (tax on share income), and the TPCVM (tax on securities sales) are now subject to IGR, as these taxes were abolished by the 2000 and 2001 Budget Laws.

(3): Agricultural income is exempt from IGR until 12/31/2010 (2001 BL). 


\begin{tabular}{|c|c|c|c|}
\hline & DESCRIPTION & DEDUCTIONS AND EXEMPTIONS & RATE \\
\hline $\begin{array}{l}\text { IGR } \\
\text { (contd.) }\end{array}$ & $\begin{array}{l}\text { B - Occupational income: } \\
\text { Definitions: } \\
\text { Occupational income includes: } \\
\text { 1-Earnings of individuals and partnerships from: } \\
\text { a -A commercial, industrial or artisanal occupation; } \\
\text { b-The profession of real estate promoter, land developer, or } \\
\text { realtor; } \\
\text { c-A liberal profession or any profession other than those } \\
\text { listed in (a) and (b). } \\
\text { 2-Income of a recurring nature, except scholarships. } \\
\text { 3- Gross earnings listed in Article } 19 \text { of the law on the IGR, } \\
\text { received by nonresident individuals or legal entities (interest } \\
\text { on loans and other fixed-income investments, royalties, } \\
\text { remuneration for technical assistance, remuneration for the } \\
\text { provision of technical and scientific information and for } \\
\text { research work, commissions and fees, etc.). } \\
\quad \text { Calculation of net occupational income: } \\
\text { Occupational income is calculated according to the real net } \\
\text { income (résultat net reel - RNR) system. } \\
\text { However, taxpayers doing business as individuals or in a de } \\
\text { facto partnership may opt for the simplified net income } \\
\text { (résultat net simplifie - RNS) system or the flat-rate system, } \\
\text { provided they meet the conditions established by law (tumover } \\
\text { (CA) thresholds). } \\
\text { 1. RNR system } \\
\text { The accounting year of taxpayers whose occupational income } \\
\text { is calculated on a real net income (RNR) basis must end on } \\
\text { December } 31 \text { of each year. }\end{array}$ & $\begin{array}{l}\text { Main exemptions: } \\
\text { (a) Total exemption for five years for enterprises } \\
\text { exporting goods or services and a } 50 \text { pcrcent reduction } \\
\text { thereafter; } \\
\text { (b) Hotels established on or after July } 1,2000 \text { are } \\
\text { entitled, for the portion of the basis of assessment } \\
\text { representing their turnover in foreign currency duly } \\
\text { repatriated directly by them or for their account by travel } \\
\text { agencies, to total exemption from IGR for the first five } \\
\text { years and a } 50 \text { percent reduction thereafter; } \\
\text { (c) } 50 \text { percent exemption with no time limit for export } \\
\text { mining enterprises and those selling their products to } \\
\text { enterprises that export them after beneficiation; } \\
\text { (d) Partial exemption of } 50 \text { percent for five years for } \\
\text { enterprises located in certain regions (1); } \\
\text { (e) Partial exemption of } 50 \text { percent for five years } \\
\text { regardless of location in Morocco for artisanal } \\
\text { enterprises; } \\
\text { (f) Partial exemption of } 50 \text { percent for five years for } \\
\text { private education and vocational training institutions. } \\
\text { The } 1998-1999 \text { budget law granted total exemption to } \\
\text { individuals performing service contracts financed by EU } \\
\text { grants, covering their occupational income under such } \\
\text { contracts. } \\
\text { Minimum taxation } \\
\text { The IGR must not be less than the minimum } \\
\text { contribution (CM) of taxpayers earning occupational } \\
\text { income subject to the RNR or the RNS system. } \\
\text { The minimum contribution is not payable during the } \\
\text { first three accounting years following the start of } \\
\text { professional activity. }\end{array}$ & $\begin{array}{l}\text { The minimum contribution } \\
\text { rates (2) are: } \\
0.25 \text { percent for the sale of the } \\
\text { following products: edible } \\
\text { oils, sugar, butter, flour, gas, } \\
\text { and petroleum products; } \\
6 \text { percent for certain liberal } \\
\text { professions (attorneys, } \\
\text { notaries, architects, engineers, } \\
\text { veterinarians, topographers, } \\
\text { etc.) } \\
0.5 \text { percent for other activities. }\end{array}$ \\
\hline
\end{tabular}

(1) Applicable to the following prefectures and provinces: Al Hoceima, Berkane, Boujdour, Chefchaouca, Es-smara, Fahs-Bai-Makada, Guelmin, Jerada, Laayone, Larache, Nador, Oued-ed-dahab, Oujda-angad, Taner-Assilah, Tan-tan, Taounate, Taourirt, Tsia, Taza, Tétouan.

(2) The basis for calculating the minimum contribution is the amount of total operating proceeds, excluding tax (2001 BL). 


\begin{tabular}{|c|c|c|}
\hline TAX & DESCRIPTION & DEDUCTIONS AND EXEMPTIONS \\
\hline $\begin{array}{l}\text { IGR } \\
\text { (contd.) }\end{array}$ & $\begin{array}{l}\text { - The RNR for each accounting year is the sum of the eamings, profits, and } \\
\text { assets obtained through the practice of one or more occupations, less } \\
\text { expenses incurred or paid. } \\
\text { To the above-mentioned earnings, profits, and assets are added inventories } \\
\text { and work in progress on the account closing date. } \\
\text { Correlatively, inventories and work in progress on the account opening } \\
\text { date are added to the expenses. } \\
\text { Inventories are assessed at cost price or at the going price if the latter is } \\
\text { lower, and work in progress is assessed at cost price. } \\
\text { A fiscal year loss may be carried forward up the fourth consecutive fiscal } \\
\text { year, except for the portion of the loss representing asset depreciation, } \\
\text { which can be carried forward indefinitely. } \\
\text { 2. RNS system } \\
\text { The RNS system is applicable optionally. } \\
\text { Simplified net income is calculated in the same way as RNR, except with } \\
\text { respect to provisions. Moreover, fiscal year losses cannot be carried } \\
\text { forward to subsequent fiscal years. } \\
\text { 3. Presumptive income system } \\
\text { The presumptive system is applicable optionally. } \\
\text { Presumptive income is calculated by applying to the turnover of each } \\
\text { calendar year a coefficient specified for each occupation. } \\
\text { The annual earnings of taxpayers opting for the presumptive income } \\
\text { system cannot be less than a minimum income comprising two elements, } \\
\text { one fixed and the other variable, determined with reference to the legal } \\
\text { bases adopted for the business license tax. } \\
\text { C. Income and profits from real estate } \\
\text { 1. Real estate income } \\
\text { - Definitions } \\
\text { The following are considered real estate income unless they fall into the } \\
\text { category of occupational income: } \\
\text { 1. income from the rental of: } \\
\text { (a) developed and undeveloped real estate and structures of all types; } \\
\text { (b) agricultural property, including constructions and fixed and mobile } \\
\text { equipment used thereon. } \\
\text { 2. Subject to the exclusions provided by law, the rental value of real estate } \\
\text { and structures that owners allow third parties to use free of charge. } \\
\text { - Calculation of net taxable income from real estate } \\
\text { Net taxable income from real estate mentioned in } 1 \text { (a) above is obtained by } \\
\text { applying a } 40 \% \text { reduction to gross real estate income. }\end{array}$ & $\begin{array}{l}\text { - Main exemptions } \\
\text { - Permanent exemptions } \\
\text { The following are exempt from the tax on the rental value of real estate that } \\
\text { owners make available free of charge to: } \\
\text { - their ascendants and descendants, when the property in question is used for } \\
\text { housing; } \\
\text { - central and local government administrations and public hospitals; } \\
\text { - designated organizations (associations reconnues d'utilite publique).. } \\
\text { - Temporary exemptions } \\
\text { Income from the rental of new constructions and structural additions is exempt } \\
\text { from the tax for the three years following the completion of such constructions. }\end{array}$ \\
\hline
\end{tabular}




\begin{tabular}{|c|c|c|c|}
\hline TAX & DESCRIPTION & DEDUCTIONS AND EXEMPTIONS & RATES \\
\hline $\begin{array}{l}\text { IGR } \\
\text { (contd.) }\end{array}$ & $\begin{array}{l}\text { 2. Real estate profits } \\
\text { Definitions } \\
\text { Real estate profits are defined as profits realized by } \\
\text { individuals upon: (1) } \\
\text { - the sale of real estatc or of rights in rem to real estate; } \\
\text { - contribution to the assets of a real estate company or of } \\
\text { rights in rem to real estate; } \\
\text { - transfer for valuable consideration or contribution to the } \\
\text { assets of a company of registered shares or partnership shares } \\
\text { issued by real estate companies, deemed neulral from a tax } \\
\text { standpoint; } \\
\text { - transfer for valuable consideration or contribution to the } \\
\text { assets of companies of shares, promoters' shares, founders' } \\
\text { shares, or partnership shares of companies engaging primarily } \\
\text { in real estate activities; } \\
\text { - grants of any kind, except for those made between } \\
\text { ascendants and descendants, between spouses, and between } \\
\text { brothers and sisters. } \\
\text { Calculation of taxable real estate profit } \\
\text { Taxable real estate profit is equal to the difference betwecn: } \\
\text { - the transfer price less transfer expenses, if any; and } \\
\text { - the purchasc price plus related costs and investment } \\
\text { expenses, this total being revalued by applying coefficients } \\
\text { established by ministerial decree, based on the national cost- } \\
\text { of-living index. }\end{array}$ & $\begin{array}{l}\text { Exempted real estate profits } \\
\text { The following are exempt: } \\
\text { Tolally } \\
\text { - Profit from the transfer of a building occupied by its owner as } \\
\text { principal residence for at least. } 10 \text { consecutive years prior to the } \\
\text { date of transfer; } \\
\text { - Profit corresponding to all or part of the transfer price, not } \\
\text { exceeding DH } 1 \text { million, of a building or part of a building } \\
\text { occupied as principal residence for more than } 5 \text { years prior to the } \\
\text { date of transfer by the owner thereof or by members of real estate } \\
\text { companies; } \\
\text { - Profit earned by individuals upon the first transfer of a building } \\
\text { used as principal residence, the area and transfer price of which do } \\
\text { not exceed } 100 \text { m }{ }^{2} \text { and DH } 200,000, \text { respectively. } \\
\text { - Profit from one or more transfers by individuals who, during the } \\
\text { calendar year, realize a total not exceeding DH } 60,000 ; \\
\text { - Profit from the transfer of undivided rights in agricultural } \\
\text { properties located outside urban areas; } \\
\text { - Between joint heirs; } \\
\text { - Between co-owners when the rights were acquired } \\
\text { more than four years earlier. } \\
\text { - A } 50 \text { percent reduction of the tax is granted on profit } \\
\text { corresponding to the portion of the transfer price exceeding the } \\
\text { limit of } D H 1 \text { million, realized on the transfer of a building } \\
\text { occupied as principal residence for } 5-10 \text { years. }\end{array}$ & $\begin{array}{l}\text { Rates applicable to real } \\
\text { estate profits } \\
\text { The rate is } 20 \text { percent. } \\
\text { However, the total } \\
\text { amount of the tax cannot } \\
\text { be less than } 3 \text { percent of } \\
\text { the transfer price. } \\
\text { This rate is in full } \\
\text { discharge of the IGR. }\end{array}$ \\
\hline
\end{tabular}




\begin{tabular}{|c|c|c|c|}
\hline TAX & DESCRIPTION & DEDUCTIONS AND EXEMPTIONS & RATES \\
\hline $\begin{array}{l}\text { IGR } \\
\text { (cont'd) }\end{array}$ & $\begin{array}{l}\text { D. Income and profits from invested capital } \\
\text { 1. Income from invested capital } \\
\text { Definitions } \\
\text { The following are considered income from invested capital: } \\
\text { - Earnings from shares or partnership shares and similar income } \\
\text { distributed by companies headquartered in Morocco and subject to } \\
\text { corporate tax (IS); } \\
\text { - Earnings from fixed-income investments deposited or credited to the } \\
\text { accounts of individuals or legal entities that have not opted for the IS and } \\
\text { whose principal residence, tax domicile, or headquarters is in Morocco. } \\
\text { Calculation of net taxable income } \\
\text { Net taxable income is calculated by deducting bank service charges and } \\
\text { collection, account maintenance, and safekeeping fees from the gross } \\
\text { amount. } \\
\text { 2. Profits from invested capital } \\
\text { Definitions } \\
\text { The following are considered profits from invested capital: annual profits } \\
\text { earned by individuals resident in Morocco upon the transfer of securities } \\
\text { and other equity and debt instruments issued by public or private legal } \\
\text { entities, whether headquartered in Morocco or abroad, as well as mutual } \\
\text { funds (OPCVM), but excluding: } \\
\text { - Companies dealing primarily in real estate; } \\
\text { - Sociétés immobilieres transparentes (real estate enterprises whose assets } \\
\text { consist of land or a housing unit owned or purchased for occupation by } \\
\text { their members). } \\
\text { Calculation of investment profits } \\
\text { Net transfer profit is calculated on the basis of the transfers of each } \\
\text { security or instrument. It is made up of the difference between: } \\
\text { - the transfer price minus, where applicable, expenses borne by the } \\
\text { transferor at the time of the transfer, particularly brokerage fees and } \\
\text { commission charges; and } \\
\text { - the purchase price plus, where applicable, expenses borne at the time of } \\
\text { any purchase, particularly brokerage fees and commission charges. }\end{array}$ & $\begin{array}{l}\text { Exempt investment profits } \\
\text { The following are exempt from IGR: } \\
\text { - Profit or the portion of profit on } \\
\text { transfers of securities and other equity } \\
\text { and debt instruments corresponding to } \\
\text { the amount of transfers made in a } \\
\text { calendar year, but not exceeding } \\
\text { DH } 20,000 \text {; } \\
\text { - Grants of securities and other equity } \\
\text { and debt instruments between } \\
\text { ascendants and descendants, between } \\
\text { spouses, and between brothers and } \\
\text { sisters. } \\
\text { Moreover, effective January } 1,2002 \\
\text { and until December } 31,2005, \text { total } \\
\text { exemption from IGR is granted for } \\
\text { profits earned from transfers of shares } \\
\text { listed on the Moroccan securities } \\
\text { exchange and from transfers of shares or } \\
\text { interests in mutual funds when at least } \\
85 \text { percent of the fund's assets are } \\
\text { continuously invested in shares listed on } \\
\text { the Moroccan securities exchange. }\end{array}$ & $\begin{array}{l}\text { Rates applicable to investment income: } \\
\text { The withholding rate is: } \\
10 \text { percent on income from shares, } \\
\text { partnership shares, and the like. This } \\
\text { withholding is in full discharge of the } \\
\text { IGR; } \\
20 \text { percent applicable to earnings from } \\
\text { fixed-income investments paid to } \\
\text { persons subject to IGR under the RNR } \\
\text { system or the RNS system. This } \\
\text { withholding is charged against the } \\
\text { amount of IGR, with entitlement to } \\
\text { refund; } \\
30 \text { percent on carnings from fixed- } \\
\text { income investments paid to individuals } \\
\text { not subject to IGR under the RNR } \\
\text { system or the RNS system. This } \\
\text { withholding is in full discharge of the } \\
\text { IGR. } \\
\text { Rates applicable to investment profits } \\
\text { l0 percent for net profits from } \\
\text { transfers of shares and other equity } \\
\text { instruments, as well as mutual fund } \\
\text { shares or interests when at least } \\
60 \text { percent of the fund's assets are } \\
\text { continuously invested in shares and } \\
\text { other equity instruments; } \\
20 \text { percent for net profits from } \\
\text { transfers of bonds and other debt } \\
\text { instruments, as well as mutual fund } \\
\text { shares or interests, when at least } \\
90 \text { percent of the fund's assets are } \\
\text { continuously invested in bonds and } \\
\text { other debt instruments; and } \\
15 \text { percent for net profits from } \\
\text { transfers of shares or interests in } \\
\text { diversified mutual funds. } \\
\text { These rates are in full discharge of the } \\
\text { IGR. }\end{array}$ \\
\hline
\end{tabular}




\begin{tabular}{|c|c|c|c|}
\hline TAX & DESCRIPTION & DEDUCTIONS AND EXEMPTIONS & RATES \\
\hline $\begin{array}{l}\text { IGR } \\
\text { (Cont'd) }\end{array}$ & $\begin{array}{l}\text { 2. Calculation of Total lncome } \\
\text { Total taxable income is the sum of } \\
\text { net incomes in the various categories } \\
\text { mentioned above. } \\
\text { The IGR is a tax for which a return } \\
\text { must be filed. } \\
\text { 3. Calculation of the Total Income of } \\
\text { Individuals who are Members of } \\
\text { Groups } \\
\text { The profits of partnerships, limited } \\
\text { partnerships, and de facto companies } \\
\text { whose members are all individuals are } \\
\text { considered occupational income of the } \\
\text { principal partner and are taxed in his } \\
\text { or her name. } \\
\text { Losses can be charged against other } \\
\text { occupational income of the principal } \\
\text { partner. }\end{array}$ & $\begin{array}{l}\text { Deductions from Total lncome } \\
\text { The following are deductible from total taxable income: } \\
\text { (a) The amount of grants in cash or in kind made to } \\
\text { designated organizations and other establishments } \\
\text { specified by law (Art. } 9 \text { of the Law on the IGR); } \\
\text { (b) Up to } 10 \text { percent of total taxable income, the amount } \\
\text { of regular interest on loans granted to taxpayers by } \\
\text { specialized institutions or by banks and credit } \\
\text { institutions for the purchase or construction of housing } \\
\text { for use as principal residence; } \\
\text { (c) Up to } 6 \text { percent of total taxable income, premiums or } \\
\text { contributions made under individual or group retirement } \\
\text { insurance contracts having a term of at least I0 years } \\
\text { concluded with insurance companies located in } \\
\text { Morocco, when the benefits are paid to recipients over } \\
50 \text { years of age. } \\
\text { Total Deductions } \\
\text { 1. Deductions for family expenses } \\
\text { The deduction is DH } 180 \text { for spouses and up to } \\
\text { DH } 1,080 \text { for dependent children. } \\
\text { 2. Deduction for life insurance contributions: } \\
\text { Taxpayers are entitled to a tax deduction of } 10 \text { percent } \\
\text { of the amount of premiums or contributions made under } \\
\text { individual or group life insurance contracts having a } \\
\text { term of at least } 10 \text { years, concluded by the insured with } \\
\text { insurance companies or cooperatives located in Morocco } \\
\text { The basis of calculation of the deduction may not } \\
\text { exceed DH } 9,000 \text { per year. }\end{array}$ & $\begin{array}{l}\text { The schedule for calculating the IGR is as } \\
\text { follows: } \\
\text { Income bracket } \\
\text { (in DH) } \\
0-20,000 \quad \text { Rate } \\
20,001-24,000 \quad \text { percent } \\
24,001-36,000 \quad 21 \text { percent } \\
36,001-60,000 \quad 35 \text { percent } \\
\text { Above } 60,000 \quad 44 \text { percent } \\
\text { However, the tax rate differs for the following } \\
\text { wages and income, for which the withholding is: } \\
\text { 10 percent for the gross income referred to in } \\
\text { Art. } 19 \text { of the law, earned by foreign enterprises } \\
\text { not domiciled or headquartered in Morocco, } \\
\text { excluding interest on loans granted in foreign } \\
\text { exchange for terms of } 10 \text { years or more; this rate } \\
\text { is in full discharge of the IGR; } \\
\text { 17 percent for substitute teachers; this } \\
\text { withholding is in full discharge of the IGR; } \\
30 \text { percent applicable to: } \\
\text { - Occasional wages and compensation paid to } \\
\text { persons who are not permanent employees of an } \\
\text { enterprise, as well as compensation paid to } \\
\text { commercial or industrial traveling salesmen or } \\
\text { representatives working for one or more } \\
\text { employers established in Morocco; this } \\
\text { withholding is not in full discharge of the IGR. } \\
\text { - Physicians working in clinics and not subject to } \\
\text { the business license tax; this withholding is in ful } \\
\text { discharge of the IGR. } \\
\text { - Gross fees paid to artists working individually } \\
\text { or in a troupe. } \\
\text { This withholding is in full discharge of the IGR. }\end{array}$ \\
\hline
\end{tabular}




\begin{tabular}{|c|c|c|c|}
\hline TAX & DESCRIPTION & DEDUCTIONS AND EXEMPTIONS & RATES \\
\hline $\begin{array}{l}\text { 2. Corporate } \\
\text { tax (impôt sur } \\
\text { les sociétés- } \\
\text { IS) }\end{array}$ & $\begin{array}{l}\text { 1. Applicability } \\
\text { Applicable by law to all corporate enterprises, } \\
\text { public institutions, and other legal entities, and } \\
\text { on an optional basis to partnerships. } \\
\text { - Taxable fiscal year earnings are the sum of } \\
\text { operating income, profits, and gains, minus } \\
\text { operating expenses. } \\
\text { - The } 1994 \text { budget law introduced the declining } \\
\text { balance method of depreciation for capital goods } \\
\text { acquired on or after January } 1,1994 \text {, on an } \\
\text { optional basis and subject to certain exclusions. } \\
\text { - Losses can be carried forward, up to the fourth } \\
\text { consecutive fiscal year; however, the portion of } \\
\text { the loss related to asset depreciation can be } \\
\text { carried forward indefinitely. } \\
\text { 2. Payment } \\
\text { - The tax is paid voluntarily in four advance } \\
\text { payments, each equal to } 25 \text { percent of the amount } \\
\text { of tax payable for the previous fiscal year. } \\
\text { - When the amount of IS due exceeds the } \\
\text { advance payments made, the outstanding balance } \\
\text { is paid voluntarily by the enterprise together with } \\
\text { the first advance payment due. } \\
\text { - If too much tax is paid, the excess is } \\
\text { automatically offset against the first advance } \\
\text { payment due and, in applicable cases, to the } \\
\text { remaining advance payments. Any balance } \\
\text { remaining is automatically refunded within a } \\
\text { month of the due date of the last advance } \\
\text { payment. }\end{array}$ & $\begin{array}{l}\text { A - Main exemptions } \\
\text { 1. Full, permanent exemption for: } \\
\text { - Nonprofit associations and comparable entities; } \\
\text { - Cooperatives and their unions, except for regional } \\
\text { mutual banks [banques régionales populaires]; } \\
\text { - Companies engaged in raising livestock; } \\
\text { - Profits on transfers of securities by foreign } \\
\text { companies. } \\
\text { - Legal entities performing service contracts financed } \\
\text { by E.U. grants pursuant to said contracts. } \\
\text { 2. Full exemption for five years and } 50 \text { percent } \\
\text { reduction thereafter for: } \\
\text { - Enterprises exporting goods } \\
\text { - Enterprises exporting services, for the portion of their } \\
\text { export turnover denominated in foreign exchange; } \\
\text { - Hotels established on or after July } 1,2000, \text { for the } \\
\text { portion of the basis of assessment corresponding to } \\
\text { their turnover denominated in foreign exchange, duly } \\
\text { repatriated directly by them or on their behalf by } \\
\text { travel agencies. } \\
\text { 3. 50 percent reduction without time limit for: } \\
\text { - Agricultural companies, for profits from grain, oil- } \\
\text { producing, sugar, fodder, and cotton crops; }{ }^{\text {;1) }} \\
\text { - Export mining enterprises, as well as those which sel } \\
\text { their products to enterprises that export them. } \\
\text { 4. 50 percent reduetion for five years for: } \\
\text { - Artisanal enterprises whose output is the result of } \\
\text { essentially manual work, whatever the location of the } \\
\text { company or enterprise; } \\
\text { - Private education and vocational training institutions } \\
\text { that began operations on or after January } 1,1998 . \\
\text { - Enterprises doing business in one of the prefectures } \\
\text { or provinces established by decree. } \\
\text { (2) }\end{array}$ & $\begin{array}{l}\text { The rate of the IS is } 35 \text { percent. } \\
\text { This rate is halved for the Tangiers area } \\
\text { (wilaya). } \\
\text { For the credit institutions, BAM, and } \\
\text { CDG, and for insurance and rcinsurance } \\
\text { companies, but not leasing companies, the } \\
\text { rate is } 39.6 \text { percent. } \\
10 \text { percent withholding at source is in } \\
\text { full discharge of the IS for: } \\
\text { - The gross earnings mentioned in Art. } 12 \text { of } \\
\text { the Law on the IS, of foreign companies, } \\
\text { excluding interest on foreign currency loans } \\
\text { granted for terms of } 10 \text { years or more; } \\
\text { interest on deposits in foreign currency or } \\
\text { convertible dirhams; interest on loans to the } \\
\text { government or guaranteed by it; as well as } \\
\text { interest on foreign currency loans granted } \\
\text { by the BRI in the context of govcrnment- } \\
\text { approved projects; } \\
\text { - Earnings from shares or partnership shares } \\
\text { and similar income ( }{ }^{3} \text { ). } \\
\text { Earnings from fixed-income investments } \\
\text { are subject to } 20 \text { percent withholding at } \\
\text { source, chargeable to IS, with the right to a } \\
\text { refund }{ }^{(3)} \\
\text { For enterprises located in export } \\
\text { processing zones, }{ }^{(4)} \text { the reduced rate of } \\
8.75 \text { percent applies to fiscal years for } \\
\text { which the deadline for reporting taxable } \\
\text { income expires on or after July } 1 \text {, 1999. } \\
\text { Foreign companies operating in Morocco } \\
\text { which have been awarded construction or } \\
\text { assembly contracts are taxed at the rate of } \\
8 \text { percent (instead of the previous } \\
12 \text { percent). } \\
\text { The } 8 \text { percent rate is calculated on turnover, } \\
\text { excluding taxes. It provides full discharge of } \\
\text { withholding at source on the gross earnings } \\
\text { of foreign companies and of tax withholding } \\
\text { on earnings from shares, partnership shares, } \\
\text { and similar income. }\end{array}$ \\
\hline
\end{tabular}

(1) Agricultural income is exempt until $12 / 31 / 2010(2001 \mathrm{BL})$.

(2) See page 2 (footnote 1 ).

(3) Withholding at source (10 percent and 20 percent) is applicable as of January 1, 2001 (BL, second half of 2000), replacing the TPA and the TPPRF.

(4) Under the $2001 \mathrm{BL}$, enterprises created on or after 1/1/2001 are fully exempt from the IS for five consecutive years as of the starting date of their operations, and the rate of 8.75 percent will apply for the following 10 fiscal years. 


\begin{tabular}{|c|c|c|c|}
\hline TAX & DESCRIPTION & $\begin{array}{l}\text { DEDUCTIONS AND EXEMPTIONS } \\
\end{array}$ & RATES \\
\hline $\begin{array}{l}\text { IS } \\
\text { (Cont'd) }\end{array}$ & & $\begin{array}{l}5 \text { - Temporary reduction for companies listed on the securities exchange } \\
\text { Eligible companies } \\
\text { Companies that list their securities on the securities exchange in a public offering } \\
\text { and/or a transfer of existing stock or a capital increase are eligible. These securities } \\
\text { must be made available to the public at the same time as the companies in question } \\
\text { are listed on the securities exchange. } \\
\text { Excluded companies } \\
\text { - Credit institutions, including finance companies; } \\
\text { - Insurance, reinsurance, and underwriting enterprises; } \\
\text { - Companies holding public utility concessions; } \\
\text { - Companies whose capital is totally or partially held by the government or a public } \\
\text { body, or by a company with at least half its capital held by a public body. } \\
\text { Rate reduction } \\
\text { Eligible companies enjoy a reduction of the IS rate applied as follows: } \\
\text { - } 25 \text { percent for companies listing thcir sccurities on the securities exchange in a } \\
\text { public offering involving the transfer of existing stock; } \\
\text { - } 50 \text { percent for companies listing their securities on the securities exchange in } \\
\text { connection with a capital increase of at least } 20 \text { percent, in which preferential } \\
\text { subscription rights are waived. } \\
\text { Effective date of the measure } \\
\text { The above reductions apply to companies listed on the securities exchange between } \\
\text { January } 1,2001 \text { and December } 31,2003 \text {. } \\
\text { The reduction will be available for three consecutive years, starting with the fiscal } \\
\text { year after the one in which the company was listed on the Casablanca Securities } \\
\text { Exchange }\end{array}$ & \\
\hline
\end{tabular}




\begin{tabular}{|c|c|c|c|}
\hline TAX & DESCRIPTION & DEDUCTIONS AND EXEMPTIONS & RATES \\
\hline $\begin{array}{l}\text { IS } \\
\text { (Cont'd) }\end{array}$ & $\begin{array}{l}\text { 3. Minimum tax } \\
\text { - The IS cannot be less than the minimum } \\
\text { contribution (cotisation minimale-CM) of DH } 1,500 \text {. } \\
\text { - The minimum contribution is not payable during the } \\
\text { first } 36 \text { months following the start of operations. } \\
\text { However, this exemption is no longer available once a } \\
\text { period of } 60 \text { months has lapsed since the date of } \\
\text { organization of the companies in question. }\end{array}$ & $\begin{array}{l}\text { Deductible items include, in particular: } \\
\text { - Purchases of materials and products; } \\
\text { - Personnel expenses; } \\
\text { - Overhead expenses; } \\
\text { - Start-up expenses; (non-amortized); } \\
\text { - Fees and taxes, except IS; } \\
\text { - Recorded depreciation; }{ }^{(1)} \\
\text { - Provisions; } \\
\text { - Grants in cash or in kind to: Habous (religious endowments); } \\
\text { designated organizations; public institutions engaged in cultural, } \\
\text { educational, or research activities; the National League Against } \\
\text { Cardiovascular Diseases; the National Cultural Action Fund; and } \\
\text { the Moroccan National Olympic Committee, there being no limit } \\
\text { on the amount of grants; } \\
\text { - Grants in cash or in kind for social projects of public or private } \\
\text { enterprises and of those institutions specified by law (up to } \\
2 \text { percent of turnover); } \\
\text { - Finance charges; } \\
\text { - Miscellaneous operating losses. } \\
\text { Up to } 20 \text { percent of the maximum depletion allowance of } \\
50 \text { percent of the taxable income of mining enterprises can be } \\
\text { allocated to a social fund and the remainder to depletion. } \\
\text { Enterprises may use all or part of the maximum investment } \\
\text { provision of } 20 \text { percent of taxable income for restructuring as well } \\
\text { as for research and development to improve their productivity and } \\
\text { economic profitability. } \\
\text { Under the } 1999 / 2000 \text { BL, enterprises can use the investment } \\
\text { provision for research and development without any limitation as to } \\
\text { amount (formerly limited to } 2 \text { percent of taxable income). }\end{array}$ & $\begin{array}{l}\text { The minimum contribution } \\
\text { rates }{ }^{(3)} \text { are: } \\
-0.25 \text { percent for } \\
\text { operations carried out by } \\
\text { business corporations and } \\
\text { involving petroleum } \\
\text { products, gas, butter, edible } \\
\text { oils, sugar, flour, water, and } \\
\text { electricity; } \\
\text { - } 0.5 \text { percent for all other } \\
\text { activities. }\end{array}$ \\
\hline
\end{tabular}

(1) The 1994 budget law instituted an optional declining balance method of depreciation for capital goods acquired on or after $1 / 1 / 1994$, subject to the exclusions specified by law.

(2) Under the 1997-1998 budget law, the time limit on using the investment provision for the coastal fishing and maritime transport sector was increascd from three years to five years.

(3) Effective January 1, 2001 (FY2000 for companies whose accounting year is the calendar year), the basis of calculation of the minimum contribution is total operating income, excluding tax (Art. 6 of the Law on the IS). 


\begin{tabular}{|c|c|c|}
\hline TAX & $\begin{array}{ll}\text { DEDUCTIONS AND EXEMPTIONS } \\
P\end{array}$ & RATES \\
\hline $\begin{array}{l}\text { IS } \\
\text { (Cont'd.) }\end{array}$ & $\begin{array}{l}\text { B-Specific reductions } \\
\text { Profits and gains realized on the transfer of fixed assets are eligible for the following reductions: } \\
\text { During operations: } \\
\text { - } 25 \text { percent if the interval between the year of acquisition of each item withdrawn from the assets } \\
\text { or transferred and the year of its withdrawal or transfer is } 2-4 \text { years; } \\
\text { - } 50 \text { percent if this interval is } 4-8 \text { years; } \\
\text { - } 70 \text { percent if this interval is more than } 8 \text { years. } \\
\text { In addition, a company enjoys total exemption if it undertakes in writing: } \\
\text { - to reinvest the total proceeds of transfers carried out in a given fiscal year no later than three } \\
\text { years after the closing date of the fiscal year in question, in capital goods or in buildings reserved } \\
\text { for the company's own business operations; and } \\
\text { - to include said goods and buildings in its assets for a period of five years, starting with the date } \\
\text { of acquisition. } \\
\text { Moreover, effective } 1 / 1 / 2002 \text { and up to } 1 / 1 / 2005 \text {, net gains and profits resulting from the } \\
\text { redemption or transfer, during operations, of shares listed on the Moroccan Securities Exchange or } \\
\text { of shares of mutual funds } 80 \text { percent of whose assets are continuously invested in shares listed on } \\
\text { the Securities Exchange, are subject separately to IS, on an optional basis, by application of a } \\
\text { reduction of } 50 \text { percent of the IS rate. } \\
\text { At the end of operations } \\
\text { - } 50 \text { percent if the interval between the year of the company's establishment and that of the } \\
\text { withdrawal or transfer of the assets is } 4-8 \text { years; } \\
\text { - Two-thirds if this interval is eight or more years. }\end{array}$ & \\
\hline
\end{tabular}




\begin{tabular}{|c|c|c|c|}
\hline TAX & DESCRIPTION & DEDUCTIONS AND EXEMPTIONS & RATES \\
\hline $\begin{array}{l}\text { Business } \\
\text { license } \\
\text { tax } \\
\text { (Impôt } \\
\text { des } \\
\text { patentes) }\end{array}$ & $\begin{array}{l}\text { The business license tax is levied on all Moroccan } \\
\text { and foreign individuals and legal entities engaged } \\
\text { in professional, industrial, or commercial } \\
\text { activities. } \\
\text { The principal amount of the business license tax } \\
\text { is a proportional tax on the rental value of } \\
\text { business premises. } \\
\text { The principal amount of the business license tax } \\
\text { is increased by a } 10 \text { percent surtax and an } \\
\text { additional percentage tax. } \\
\text { The principal amount of the business license tax } \\
\text { is allocated as follows: } \\
\text { - } 90 \text { percent for local governments; } \\
\text { - } 10 \text { percent for the general state budget. } \\
\text { The additional percentage tax is earmarked for the } \\
\text { general state budget and the } 10 \text { percent surtax is } \\
\text { paid to boards of trade, industry, marine fisheries, } \\
\text { and crafts and their federations. } \\
\text { Basis of assessment of the TP: } \\
\text { The proportional tax (TP) is assessed on the } \\
\text { current, regular, gross rental value of stores, } \\
\text { factories, workshops, sheds, yards, and places of } \\
\text { deposit and of premises, sites, and facilities used } \\
\text { for taxable business activities. }\end{array}$ & $\begin{array}{l}\text { Main exemptions: } \\
\text { The following are exempt from the business } \\
\text { license tax: } \\
\text { - Civil servants; } \\
\text { - Individuals in one of the occupations listed in } \\
\text { Category 7, Table A; } \\
\text { - Farmers, but only for the sale (other than in } \\
\text { shops or stores) of crops and products from } \\
\text { their farm, as well as the sale of livestock they } \\
\text { raise there; } \\
\text { - Mutual funds (OPCVMs) in accordance with } \\
\text { the applicable legislation; } \\
\text { - Individuals and legal entities engaged in } \\
\text { professional, industrial, or business activities, } \\
\text { for their first five years of operation. }{ }^{(1)} \\
\text { However, this exemption does not apply to: } \\
\text { The credit institutions, BAM, and CDG; } \\
\text { Insurance and reinsurance companies; } \\
\text { Real estate agencies. } \\
\text { Reduction: To determine the rental value to be } \\
\text { used as the base for TP payable by hotels, the } \\
\text { following reductions are applied to the cost } \\
\text { price of the buildings and facilities of each } \\
\text { establishment: } \\
\text { - } 20 \text { percent for cost prices of DH } 3 \text { million or } \\
\text { less; } \\
\text { - } 40 \text { percent for cost prices of DH } 3-6 \text { million; } \\
\text { - } 50 \text { percent for cost prices of DH } 6-12 \text { million; } \\
\text { and } \\
\text { - } 60 \text { percent for cost prices of over DH } \\
12 \text { million. }\end{array}$ & $\begin{array}{l}\text { Applicable rates: } \\
\text { Rental value, which is the basis of calculation } \\
\text { of the business license tax for factories, } \\
\text { industrial and commercial establishments, and } \\
\text { service providers, is determined directly by } \\
\text { applying a flat rate of } 3 \text { percent ( } 2003 B L \text { ) to } \\
\text { the cost price of land, constructions, fittings, } \\
\text { equipment, and tools. } \\
\text { The rental value used as the basis of } \\
\text { calculation of the TP payable on land, } \\
\text { buildings and their fittings, equipment, and } \\
\text { tools is limited to the portion of their cost } \\
\text { price not exceeding DH 50 million } \\
\text { (DH } 100 \text { previously) (BL, second half of } \\
2000 \text { ). } \\
\text { This restriction applies to all establishments } \\
\text { producing goods and engaging in the } \\
\text { manufacture, processing, or packaging of } \\
\text { products. } \\
\text { The cap on the taxable amount of an } \\
\text { investment also applies to service providers } \\
\text { ( } 2001 \text { BL). }\end{array}$ \\
\hline
\end{tabular}

N.B. (1) The Budget Law for the second half of 2000 extended the five-year exemption to the 10 percent surtax and the additional percentage tax.

(2) In the interest of harmonization, the 1997-1998 BL made contract managers also eligible for the reduction granted to owner-operated hotels. 


\begin{tabular}{|c|c|c|c|}
\hline TAX & DESCRIPTION & DEDUCTIONS AND EXEMPTIONS & RATES \\
\hline $\begin{array}{l}\text { 4. Urban } \\
\text { tax (Taxe } \\
\text { urbaine-- } \\
\text { TU) }\end{array}$ & $\begin{array}{l}\text { The urban tax is levied on: } \\
\text { - Buildings and constructions of all types, occupied fully } \\
\text { or in part by their owners as their principal or secondary } \\
\text { residence, or provided free of charge by said owners as } \\
\text { housing for their spouse, ascendants, or descendants; } \\
\text { - Buildings used by their owners for a business activity } \\
\text { or any other type of operation; } \\
\text { - Machinery and equipment installed in enterprises that } \\
\text { produce goods or services; } \\
\text { - Undeveloped land used by the owner for any type of } \\
\text { operation. } \\
\text { TU is levied within the boundaries of urban communes } \\
\text { and their outlying areas, designated centers, summer } \\
\text { and winter resorts, and thermal spas. } \\
\text { TU is assessed on the rental value of land, buildings, } \\
\text { machinery, and equipment, as determined by the survey } \\
\text { committee, using comparisons or direct valuation. } \\
\text { However, a } 75 \text { percent reduction is applicable to buildings } \\
\text { occupied as by the owner as his or her principal residence } \\
\text { or free of charge by the owner's ascendants or descendants. } \\
\text { Effective January } 1,2002 \text {, the rental value is increased } \\
\text { annually by } 2 \text { percent. } \\
\text { Ninety percent of TU revenue is allocated to communes } \\
\text { and } 10 \text { percent to the general state budget to cover } \\
\text { assessment and collection costs. }\end{array}$ & $\begin{array}{l}\text { Permanent exemptions: } \\
\text { - Royal residences, properties owned by the state, local } \\
\text { governments, private relief and charity organizations, } \\
\text { and designated organizations; } \\
\text { - Endowed property [biens habous], with the exception of } \\
\text { endowed property involving households; } \\
\text { - Buildings made available free of charge to certain } \\
\text { organizations specified by law; } \\
\text { - Buildings owned by foreign governments and } \\
\text { designated for use as offices or housing for certain } \\
\text { diplomats (subject to reciprocity); } \\
\text { - Buildings owned by international organizations } \\
\text { enjoying diplomatic status and designated for use as } \\
\text { offices of the organization or as housing for mission } \\
\text { chiefs; } \\
\text { Buildings generating no income and used exclusively } \\
\text { for public religious services or free classes, and } \\
\text { buildings classified as historical monuments; } \\
\text { Buildings owned by agricultural cooperatives and their } \\
\text { unions, designated for agricultural use. } \\
\text { Temporary exemptions: } \\
\text { Five years for: } \\
\text { - Newly-installed machinery and equipment; } \\
\text { - New construction and structural additions; } \\
\text { - New construction and structural additions as well as } \\
\text { capital goods, materials, and tools to be used solely for } \\
\text { business purposes when acquired by leasing companies } \\
\text { on behalf of their customers, not including those owned } \\
\text { by: } \\
\text { - Long-term establishments of companies and } \\
\text { enterprises not headquartered in Morocco that have } \\
\text { been awarded contracts for construction work, supplies, } \\
\text { or services; } \\
\text { - The credit institutions, BAM, and CDG; } \\
\text { - Insurance and reinsurance enterprises; } \\
\text { - Real estate agencies. } \\
\text { 15 years for: } \\
\text { Dwellings considered low-cost housing for the full term } \\
\text { of loans, although this exemption may not exceed } \\
\text { 15 years from the date of their completion. }\end{array}$ & 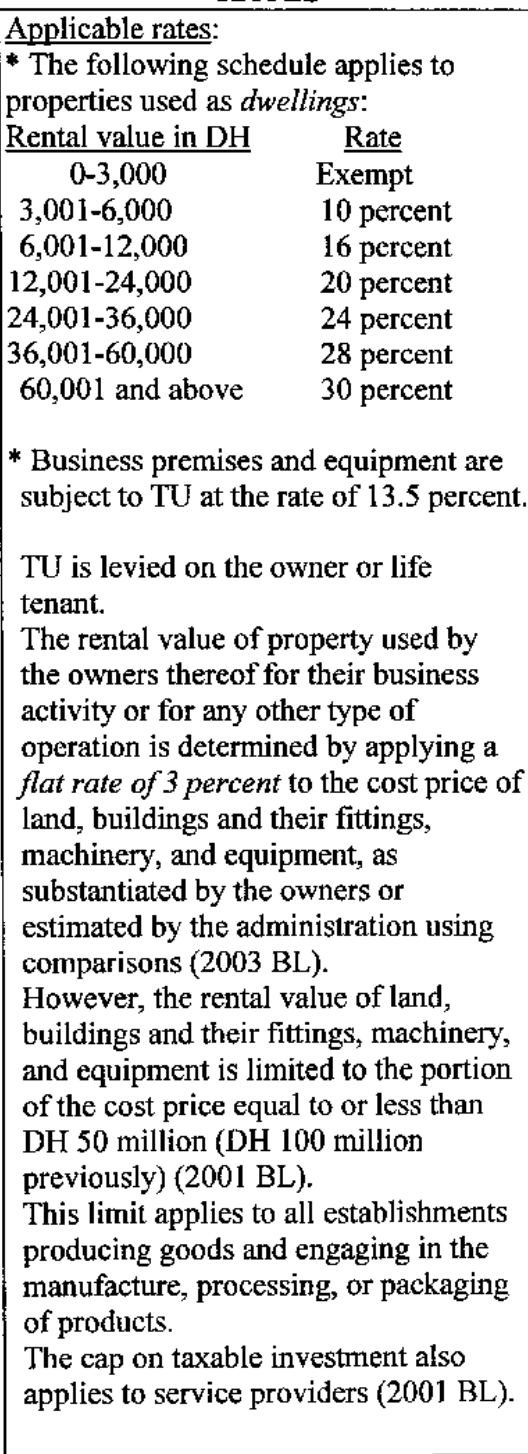 \\
\hline
\end{tabular}




\begin{tabular}{|c|c|c|c|}
\hline TAX & \begin{tabular}{|c|} 
DESCRIPTION \\
\end{tabular} & DEDUCTIONS AND EXEMPTIONS & RATES \\
\hline $\begin{array}{l}\text { 5. Munici- } \\
\text { pal tax } \\
\text { (Taxe } \\
\text { d'édilité- } \\
\text { TE) }\end{array}$ & $\begin{array}{l}\text { The TE applies to buildings and constructions of all types, land used } \\
\text { for any kind of operation, and machinery and equipment subject to } \\
\text { the urban tax. } \\
\text { The tax is based on the rental value used to calculate the urban tax, } \\
\text { including that of buildings which are temporarily exempt, and on the } \\
\text { total amount of rent when the property is leased. } \\
\text { All TE revenue is earmarked for the communes. }\end{array}$ & $\begin{array}{l}\text { Exemptions: } \\
\text { Buildings and building annexes permanently } \\
\text { exempt from the urban tax are also exempt } \\
\text { from the TE. }\end{array}$ & $\begin{array}{l}\text { Applicable rates: } \\
\text { The rates of the TE are: } \\
\text { - } 10 \text { percent for buildings located } \\
\text { within the boundaries of urban } \\
\text { communes and designated } \\
\text { centers; } \\
\text { - } 6 \text { percent for buildings located } \\
\text { in the outlying districts of } \\
\text { urban communes. }\end{array}$ \\
\hline
\end{tabular}




\begin{tabular}{|c|c|c|c|}
\hline TAX & DESCRIPTION & \begin{tabular}{|l} 
DEDUCTIONS AND EXEMPTIONS \\
\end{tabular} & RATES \\
\hline $\begin{array}{l}\text { II. Value- } \\
\text { added tax } \\
\text { (VAT) }\end{array}$ & $\begin{array}{l}\text { 1/ Applicability } \\
\text { VAT applies to industrial, } \\
\text { artisanal, and commercial } \\
\text { activities (including retail } \\
\text { businesses with an annual } \\
\text { turnover of DH } 2,000,000 \text { or } \\
\text { more), professional occupations, } \\
\text { and import operations. } \\
\text { 2/ Basis of assessment: } \\
\text { - Taxable turnover includes the } \\
\text { price of goods, works, or } \\
\text { services and income incidental } \\
\text { thereto, as well as related fees, } \\
\text { taxes, and duties, with the } \\
\text { exception of VAT. } \\
\text { - Turnover consists of: } \\
\text { - for the operations of real estate } \\
\text { developers, the transfer price of } \\
\text { the construction minus the price } \\
\text { of the land, adjusted with } \\
\text { reference to real estate profit } \\
\text { ratios. However, when the } \\
\text { purpose of the building is other } \\
\text { than for sale, the basis of } \\
\text { assessment is the cost price of the } \\
\text { construction; } \\
\text { - for land development } \\
\text { operations, the cost of the work; } \\
\text { - for swap and self-delivery } \\
\text { operations, the regular price of the } \\
\text { goods, works, or services at the } \\
\text { time the operations are carried } \\
\text { out. } \\
3 / \text { Returns } \\
\text { Two types of returns are } \\
\text { prescribed by law: } \\
\text { - Monthly: } \\
\text { - for taxpayers whose taxable } \\
\text { turnover during the year is } \\
\text { DH } 2,000,000 \text { or more } \\
\text { - for any person without a long- } \\
\text { term establishment in Morocco } \\
\text { who engages in taxable } \\
\text { operations in the country. } \\
\text { - Ouarterly: }\end{array}$ & $\begin{array}{l}\text { There are two types of exemption: } \\
\text { 1. Exemptions without entitlement to deduction (sans droit à déduction-SDD) } \\
\text { These apply, in particular, to: essential goods, products subject to price controls } \\
\text { books and newspapers, sales of and services provided by manufacturers or } \\
\text { suppliers with a turnover of DH } 180,000 \text { or less, self-delivery of dwellings } \\
\text { having an area of } 240 \mathrm{~m}^{2} \text { or less, operations and interests involving advances } \\
\text { and loans to the central and local governments, etc. } \\
\text { The following are also exempt: } \\
\text { - Medical treatment provided by physicians, dentists, physical therapists, } \\
\text { orthopedic specialists, speech therapists, and midwives. } \\
\text { Moreover, effective } 1 / 1 / 2002 \text {, the operators of clinics, health or treatment } \\
\text { centers, and medical laboratories are exempt without entitlement to deduction; } \\
\text { - Interest on loans granted by finance companies, on the same terms as those } \\
\text { granted to private school students and vocational training by banks; } \\
\text { - Sales of equipment specially made for the handicapped, locally-produced } \\
\text { handcrafted carpets, and operations related to loans and advances to the } \\
\text { Communal Capital Fund (Fonds d'Équipement Communal), etc. } \\
\text { With effect from } 1 / 1 / 2001 \text {, the same entitlement to VAT refunds granted to } \\
\text { diplomatic missions is also applicable to international and regional } \\
\text { organizations and their members accredited to Morocco and having diplomatic } \\
\text { status. } \\
\text { 2. Exemptions with entitlement to deduction (avec droit à deduction-ADD) } \\
\text { These apply essentially to products delivered and services provided for taxpayes } \\
\text { export activities, fertilizers, agricultural equipment, low-cost housing having an } \\
\text { area and a VIT (total real estate value) of } 100 m^{2} \text { and } D H 200,000, \text { respectively, } \\
\text { and ship sales, repairs, and conversions. } \\
\text { - Capital goods to be recorded in a fixed assets account, acquired by taxpayers } \\
\text { - Capital goods acquired by private education and vocational training } \\
\text { institutions; } \\
\text { - New vehicles acquired by taxi operators; } \\
\text { - Capital goods acquired by the civil defense department; } \\
\text { - Goods and services required for foreign film production. }\end{array}$ & $\begin{array}{l}\text { Applicable rates: } \\
\text { 1. Four ad valorem rates: } \\
\text { - Regular rate: } 20 \text { percent; } \\
\text { - Rate of } 14 \text { percent: } \\
\text { ADD: for real estate projects, coffee, } \\
\text { tea, economical light commercial } \\
\text { vehicles, economical mopeds, etc. } \\
\text { - SDD: for services provided by } \\
\text { insurance agents and brokers; } \\
\text { - Rate of } 10 \text { percent, ADD, applicable to } \\
\text { the tourism sector. } \\
\text { Effective January } 1,2001 \text {, the following } \\
\text { are also subject to the } 10 \text { percent rate: } \\
\text { - Food services (fast-food restaurants, } \\
\text { caterers, etc); } \\
\text { - Sales of foodstuffs and beverages for on- } \\
\text { site consumption in restaurants, regardless } \\
\text { of location; } \\
\text { - Food service operations by providers of } \\
\text { services to employees of businesses. } \\
\text { - A reduced rate of } 7 \text { percent: } \\
\text { - SDD: for certain professional occupations } \\
\text { (atlorncys, notarics, veterinarians, } \\
\text { interpreters, etc.); } \\
\text { - ADD: } \\
\text { - For certain mass consumption goods } \\
\text { (water, electricity, pharmaceuticals, etc.); } \\
\text { - For economical vehicles; bank, credit, } \\
\text { and exchange operations; securities } \\
\text { transactions carried out by brokerage } \\
\text { firms; and transactions involving shares } \\
\text { and partnership shares issued by mutual } \\
\text { funds. }\end{array}$ \\
\hline
\end{tabular}




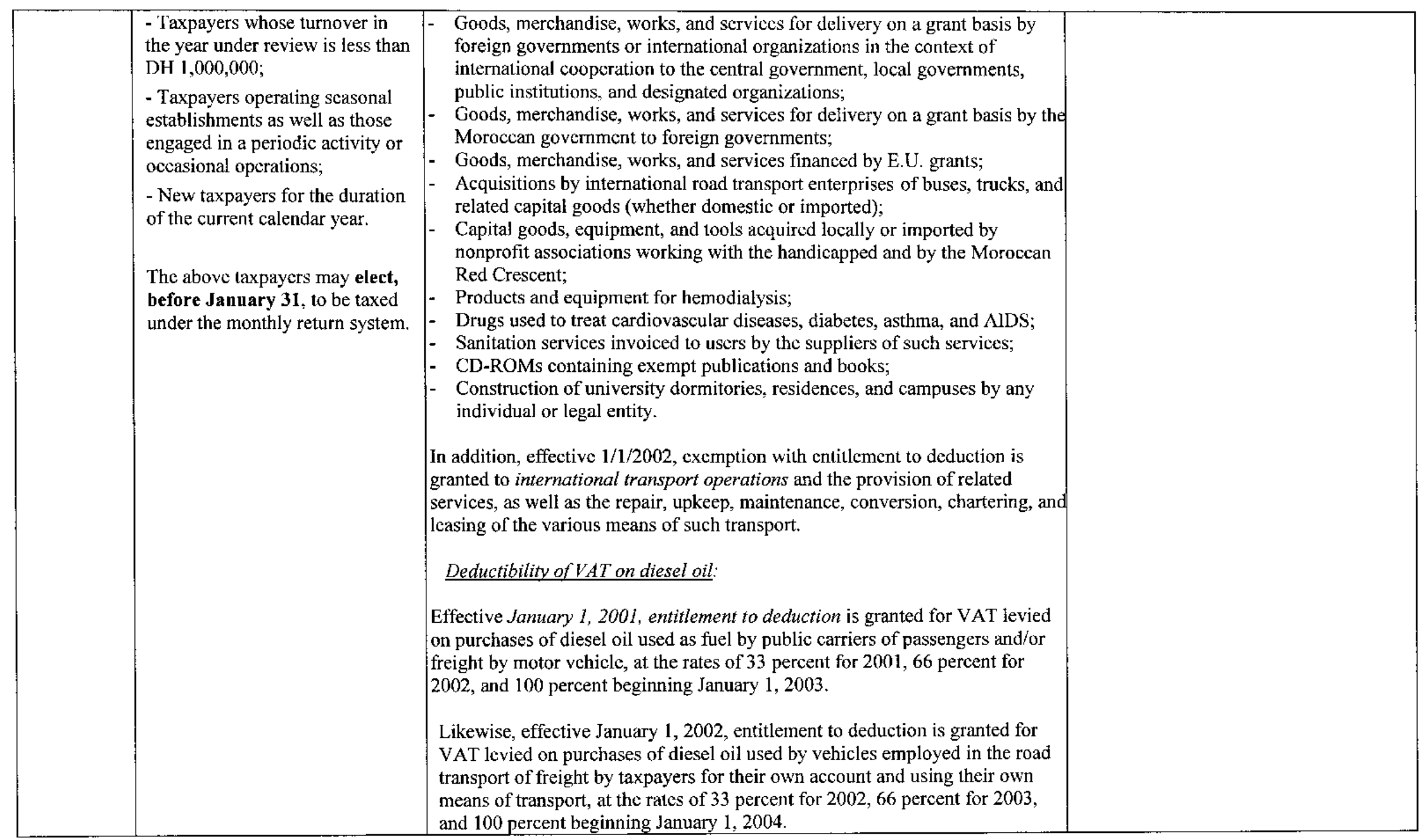




\begin{tabular}{|c|c|c|c|}
\hline TAX & DESCRIPTION & DEDUCTIONS AND EXEMPTIONS & RATES \\
\hline $\begin{array}{l}\text { III. Regis- } \\
\text { tration } \\
\text { fees and } \\
\text { stamp } \\
\text { duty } \\
\text { 1. Regis- } \\
\text { tration } \\
\text { fees (DE) }\end{array}$ & $\begin{array}{l}\text { There are two types of registration fees: fixed } \\
\text { and proportional fees. } \\
\text { The main operations to which these fees apply } \\
\text { obligatorily are: } \\
\text { - Transfers inter vivos, with or without } \\
\text { payment, of property and rights in rem to real } \\
\text { estate; } \\
\text { - Long-term leases of real estate companies; } \\
\text { - Transfers of shares in real estate companies } \\
\text { or partnership shares, interests in partnerships. } \\
\text { - Effective } 1 / 1 / 2002 \text {, documents pertaining to } \\
\text { groupements d'interêt économique (GIE) } \\
\text { (cooperatives). }\end{array}$ & $\begin{array}{l}\text { Main exemptions: } \\
\text { 1 - Registration fees: } \\
\text { All documents drawn up in the public interest are exempt } \\
\text { from the registration requirement. } \\
\text { The following are also exempt from registration fees: } \\
\text { - Public accounting documents; } \\
\text { - Mortgage loan operations between individuals and credit } \\
\text { institutions; } \\
\text { - Real estate leasing operations; } \\
\text { - acquisition of land for the execution of investment } \\
\text { projects within a maximum period of } 36 \text { months. } \\
\text { Documents for the acquisition of buildings necessary for } \\
\text { accomplishment of the purpose of nonprofit associations } \\
\text { working with the handicapped are also exempt from } \\
\text { registration fees. } \\
\text { 2. Stamp duties: } \\
\text { The following are exempt from the stamp duty } \\
\text { requirement: } \\
\text { - Government securities and bonds; } \\
\text { - Instruments and documents drawn up in the public } \\
\text { intercst. } \\
\text { - Passenger tickets and bills of lading. } \\
\text { offices are exempt from the DH } 20 \text { stamp duty. }\end{array}$ & $\begin{array}{l}\text { Stamp duties: } \\
\text { There are three categories: } \\
\text { Size stamp: DH } 20 \text { rate. } \\
\text { Stamp duty of DH } 20 \text { is payable on each sheet of } \\
\text { paper used, regardless of size } \\
\text { All documents and instruments, whether public } \\
\text { or private, books, registers, directories, letters, } \\
\text { extracts, copies, authenticated copies, } \\
\text { photocopies, and any other reproductions of } \\
\text { these documents obtained by photographic or } \\
\text { other means which must or can be produced in } \\
\text { court or submitted to the authorities by way of } \\
\text { obligation, release, proof, pleading, or defense. } \\
\text { - Proportional stamp: paid in proportion to } \\
\text { the amount listed on the securities; } \\
\text { - Special stamp: affixed to passports, receipts, } \\
\text { etc. } \\
\text { The } 1997-1998 \text { BL reduced the stamp duty: } \\
\text { - from } 10 \text { percent to } 5 \text { percent on television } \\
\text { advertisements. }\end{array}$ \\
\hline
\end{tabular}




\begin{tabular}{|c|c|c|}
\hline TAX & DESCRIPTION & RATES \\
\hline $\begin{array}{l}\text { Stamp duty } \\
\text { (Cont'd) }\end{array}$ & & $\begin{array}{l}\text { The stamp duty is DH } 20 \text { for: } \\
\text { - Bills of lading; } \\
\text { - Individual maritime logs; } \\
\text { - Anthropometric records [fiches anthropométriques]; } \\
\text { - Copies of affidavits concerning vehicle accidents. } \\
\text { The rates for receipts and releases are revised as follows: } \\
\text { - Up to DH 10: exempt; } \\
\text { - DH } 10 \text {-DH 100: DH } 0.50 \text {; } \\
\text { - DH } 100-\mathrm{DH} 500 \text { : DH } 2.50 \text {; } \\
\text { - DH } 500 \text { and above per bracket or sub-bracket: DH } 1.25 \text {; } \\
\text { - For documents representing unconditional receipt and merchandise shipping receipts: DH } 1.25 \text {. } \\
\text { Commercial paper which, at the time of issue, circulation, or presentation in Morocco, indicates } \\
\text { domiciliation at a credit institution or postal checking office is subject to a fixed stamp duty of DH } 5 \text {. } \\
\text { The following are subject to: } \\
\text { - Fixed duty of DH } 300 \text { : } \\
\text { - Documents related to credit transactions between finance companies and individuals; } \\
\text { - Documents establishing or canceling mortgages and charges on a business granted as backing for said } \\
\text { transactions. } \\
\text { - Transfer duty of } 5 \text { percent (10 percent previously): transfers of business and of the clientele of hotels, } \\
\text { restaurants, establishments selling alcoholic beverages, and cinemas. }\end{array}$ \\
\hline
\end{tabular}




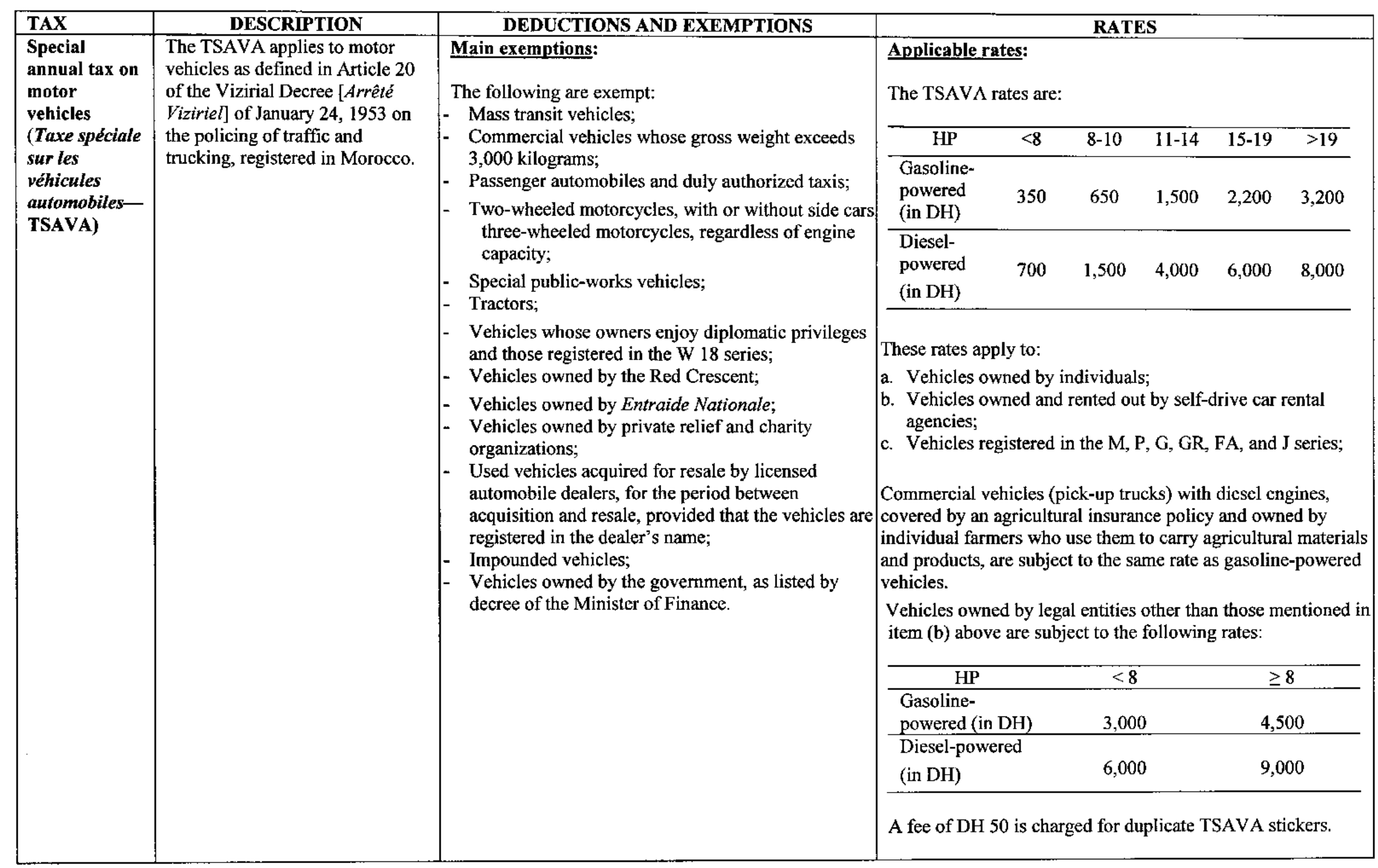




\begin{tabular}{|c|c|c|c|}
\hline TAX & DESCRIPTION & DEDUCTIONS AND EXEMPTIONS & RATES \\
\hline $\begin{array}{l}\text { Tax on } \\
\text { insurance }\end{array}$ & $\begin{array}{l}\text { The tax on insurance applies to insurance policies } \\
\text { concluded by insurance companies as well as any } \\
\text { document whose sole purpose is to draw up, } \\
\text { modify, or amicably cancel such contracts, } \\
\text { excluding stamp duty, registration fees, and value- } \\
\text { added tax. } \\
\text { The tax is assessed on the amount of premiums, } \\
\text { surcharges, or contributions. } \\
\text { It is paid in quarterly advance payments by } \\
\text { insurance companies. }\end{array}$ & $\begin{array}{l}\text { Main exemptions: } \\
\text { Insurance contracts covering the following are } \\
\text { exempt: } \\
\text { - Occupational accidents and illnesses; } \\
\text { - Agricultural mutual insurance; } \\
\text { - Coverage for war risks; } \\
\text { - Payments made to the Moroccan retirement fund; } \\
\text { - Life insurance; } \\
\text { The tax is not payable on life insurance policies } \\
\text { or life annuities taken out by persons not } \\
\text { domiciled or normally resident in Morocco, or on } \\
\text { any other policies when the risk is centered } \\
\text { abroad or pertains to an establishment located } \\
\text { there. }\end{array}$ & $\begin{array}{l}\text { Applicable rates: } \\
\text { The rates of the tax on insurance are } \\
3 \text { percent, } 6 \text { percent, or } 12 \text { percent, } \\
\text { depending on the type of insurance policy. } \\
\text { The } 1998-1999 \text { BL introduced an } \\
\text { administrative fine of DH } 500 \text { per day of } \\
\text { lateness (previously DH } 50 \text { ), applicable to } \\
\text { insurance companies that fail to produce } \\
\text { documents or publications prescribed by law } \\
\text { within the specified period. }\end{array}$ \\
\hline
\end{tabular}

